ÉGYPTE monde arabe

\section{Égypte/Monde arabe}

23 | 1995

Géographies de l'Égypte 2

\title{
Le Caire : aspects sociaux de l'étalement urbain
}

Entre spécialisation et mixité

\section{Eric Denis}

\section{(2) OpenEdition}

Journals

Édition électronique

URL : https://journals.openedition.org/ema/968

DOI : $10.4000 /$ ema.968

ISSN : 2090-7273

Éditeur

CEDEJ - Centre d'études et de documentation économiques juridiques et sociales

Édition imprimée

Date de publication : 30 septembre 1995

Pagination : 77-130

ISSN : 1110-5097

Référence électronique

Eric Denis, "Le Caire : aspects sociaux de l'étalement urbain », Égypte/Monde arabe [En ligne], 23 |

1995, mis en ligne le 08 juillet 2008, consulté le 07 juillet 2022. URL : http://journals.openedition.org/ ema/968; DOI : https://doi.org/10.4000/ema.968

Ce document a été généré automatiquement le 7 juillet 2022

Tous droits réservés 


\section{Le Caire : aspects sociaux de l'étalement urbain}

Entre spécialisation et mixité

\section{Eric Denis}

1 «Chez le voyageur qui la contemplait pour la première fois, la ville éveillait le désir d'une comparaison, mais il s'apercevait aussitôt que c'était un piège, car elle les rejetait toutes; elle ne ressemblait en effet à rien. $»^{1}$ Le Caire pourrait être cette ville tant elle semble échapper aux rapprochements. Sa complexité semble irréductible et ne renvoie à rien de comparable. «Le Caire n'est pas un roman, c'est un conte circulaire qui va dans tous les sens. $»^{2}$

2 Le caractère à la fois inextricable et irréductible de la totalité cairote n'apparaît pas uniquement dans les approches littéraires. Quand elles cherchent à rendre compte des changements qui accompagnent la "massification» du Caire, les sciences sociales développent des idées analogues. La transition à marche forcée d'une aire métropolisée de 2,5 millions d'habitants dans les années cinquante, à la mégapole d'environ 11 millions d'habitants de la fin des années quatre-vingt-dix, s'est traduite par une multiplication des modèles qui tentent de rendre compte de la bigarrure des paysages. Tout a bougé, s'est recomposé autour de combinaisons de plus en plus complexes supportant mal les synthèses en quelques catégories bien assurées.

3 Pour Philippe Haeringer ${ }^{3}$, le propre d'une mégapole est de ne pas se laisser dessiner. «La cartographie s'essouffle à suivre l'extension » du Caire, cette « énorme ville », note André Raymond ${ }^{4}$. Définir ses limites ou sa forme se révèle une tâche par avance vouée à l'échec. Depuis longtemps la ville a échappé au paradigme de l'enceinte. Le Caire contemporain est proprement inclôturable.

Tableau 1 : L'agglomération du Caire*

\begin{tabular}{|l|l|l|l|l}
\hline $\begin{array}{l}\text { Le Caire (pop. en } \\
\text { milliers) }\end{array}$ & $\begin{array}{l}\text { Le Caire (taux annuel de } \\
\text { croissance \%) }\end{array}$ & $\begin{array}{l}\text { Égyte (taux annuel de } \\
\text { croissance \%) }\end{array}$ \\
\hline
\end{tabular}




\begin{tabular}{|l|l|l|l|}
\hline 1947 & 2.568 & - & - \\
\hline 1960 & 4250 & 3,95 & 2,45 \\
\hline 1966 & 5.495 & 4,38 & 2,47 \\
\hline 1976 & 7.184 & 2,72 & 2,02 \\
\hline 1986 & 9.487 & 2,82 & 2,75 \\
\hline 1990 & $10.330^{* *}$ & 2,15 & 2,38 \\
\hline 1994 & $11.052^{* * *}$ & 1,93 & 2,27 \\
\hline
\end{tabular}

* Agglomération morphologique, défınition Géopolis (continuité du bâti < 200 m).

** Projection F. Moriconi.

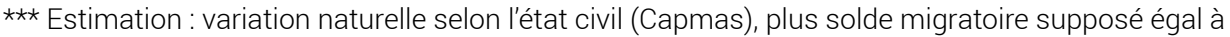
celui observé entre 1976 et 1986 (62.000 migrants par an en faveur de cette zone) - taux de croissance annuel, période 1986-1994.

4 Tout aussi difficilese révèle le projet de caractériser la composition intra-urbaine du Caire. Cela suppose comme une mise en abîme. Décrire la ville implique d'avoir recours à des échelles toujours plus petites : la ville appelle le quartier; le quartier, la rue et la place; la rue, l'immeuble; et l'immeuble, le logement. Rien ne semble réductible à quelques profils. Galila el Kadi souligne qu'« alors même que les écarts entre riches el pauvres demeurent considérables (...), les différences ne se traduisent pas aussi clairement dans l'espace et la construction $»^{5}$. De la même façon, Robert Ilbert insiste sur l'importance et la puissance des mouvements internes qui réduisent à néant les délimitations assurées: "Cela signifie que vous ne trouverez plus un seul quartier vraiment résidentiel et luxueux, non plus d'ailleurs qu'une seule zone qui soit un véritable bidonville. ${ }^{6}$ Dimension également soulignée par Mercedes Volait : " Ainsi, à la disparité - bien réelle, cette dernière - des revenus, correspondrait désormais une sorte d'homogénéité spatiale. ${ }^{7} L^{\prime}$ 'analyse des arrangements socio-spatiaux ne serait donc plus heuristique : trop grossière, elle ne permettrait pas de mettre en évidence les différences qui produisent désormais la mosaïque urbaine.

5 Dans nos essais de caractérisation de cette accumulation sociale, économique et politique qui constitue la ville, nous ne pouvons ignorer ou gommer ces subtilités. Il existe comme une fluidité du modèle cairote, comme une propriété qui le conduit à ne pas supporter les découpages. Comme si, la mégapole étant en permanence agitée de vibrations, le cliché était toujours flou. De plus, elle déborde du cadre. Elle s'étale. Le Caire ne pourrait donc se lire qu'à contre-courant des approches qui définissent des secteurs homogènes et qui découpent la ville en confondant morphologie urbaine et contenu social ${ }^{8}$.

\section{Donner à voir des dynamiques socio-spatiales}

6 La totalité cairote ne déroge pas à ce qui apparaît comme une règle : «Par définition, les espaces urbains sont flous et ductiles. II serait donc vain de prétendre à des découpages territoriaux définitifs. $»^{9}$ Dansla mégapole rien n'est homogène, tout 
change, tout s'imbrique et se déplace, de même que tout est en relation, interdépendant. Mais en disant cela nous ne faisons que redécouvrir de manière presque naïve la complexité, dimension consubstantielle d'une science sociale se refusant à la simplification véhiculée parle sens commun et les médias.

II reste indispensable de dire où cela se passe: il s'agit toujours d'une méthode d'objectivation fondamentale, manière irremplaçable de dire les différences. En outre, comme l'a montré Alfredo Mela ${ }^{10}$, ce qui est réseau à une échelle peut très bien devenir forme et classe unique à une échelle plus petite. En ce qui concerne l'approche du fait urbain, cela revient, bien sûr, à considérer l'emboîtement des échelles et les interactions spatiales comme deux dimensions indissociables des édifices sociaux et de leurs inscriptions matérielles. Toutefois, cela peut aussi amener à penser que les échelles les plus petites sont trop réductrices, qu'elles ne sont que des masques et que tout se passe ou ne peut se voir que du quartier ou de la rue, le reste n'étant que raccourcis et réductions. Seulement, sans la médiation des échelles supérieures, la spécificité dégagée pourrait bien n'être qu'une focalisation empirique ou un épiphénomène pris pour une dimension structurelle. Notre analyse aurait dès lors une tournure de "pensée faible " survalorisant des dimensions qualifiées de culturelles ou d'identitaires comme pour mieux gommer des changements structuraux ou des inégalités ${ }^{11}$.

8 En retenant, comme grille de lecture de la dynamique cairote, l'axiome a priori simpliste mais incontournable - diversité plus densité égale urbanité - les contours d'un produit social sont en mesure de s'animer.Il ne s'agira plus d'un type régional, mais d'une figure du phénomène le plus universel qui soit, à savoir la métropolisation du monde; un produit naturellement complexe, plein de résistances et d'irréductibilités qui fondent l'intérêt de son étude.

Pour mener à bien cette mesure de la distribution des groupes sociaux et des tris sociaux, nous utiliserons la distribution en sept catégories socio-professionnelles proposées par les recensements de 1976 et $1986^{12}$. Nous montrerons la manière dont elles se distribuent et indiquerons quelques tendances quant à l'évolution du rapport entre distance spatiale et distance sociale. La croissance et l'étalement, «la massification", n'auraient-ils pas comme effet de remettre en cause la diversité citadine, c'est-à-dire la mixité sociale des quartiers? Autrement dit, nous essaierons de proposer, au moins à l'échelle de l'agglomération découpée en quartiers, une mise en évidence des structures socio-spatiales et de leurs redistributions.

La mixité et la proximité entre des populations différentes peuvent être retenues comme des dimensions universelles spécifiques de l'urbanité, c'est-à-dire un produit social disposant d'un puissant potentiel d'évolution au regard d'un contre-type rural centré sur la communauté agricole et où, donc, en l'absence d'un potentiel d'altérité, le changement peut moins aisément se produire ${ }^{13}$. Mais nous verrons aussi que, selon une logique de continuum, cette dichotomie n'est plus aussi certaine. En raison de la multiplication des flux matériels et immatériels qui déforment les distances et les dépendances, les frontières entre ruralité et urbanité sont en effet plus floues. En d'autres termes, la diffusion des valeurs qualifiées d'urbaines précède les formes urbaines physiques, matérielles, voire n'implique pas ces dernières. Par exemple, les activités professionnelles se diversifient, se détachent de l'agriculture, sans que l'aspect des villages concernés s'en trouve radicalement modifié. 
11 Inversement, à l'intérieur de la ville elle-même, dans ce contexte d'étalement et de transition de la métropole vers la mégapole, nous observerons que la mixité sociale est en partie remise en cause. Elle est fractionnée, décalée ou décentrée. Pour le moins, apparaît clairement une inversion du modèle d'urbanisation. Désormais, il est moins altéré par un flux toujours plus faible de ruraux attirés par les lumières de la ville que par le déversement de la condition urbaine dans les sociétés rurales. Cela vaut tant pour les périphéries de la capitale que pour l'ensemble d'un étroit écoumène égyptien jamais très loin de son système de villes.

\section{Des matériaux pour l'approche de la diversitéurbaine}

\section{Sept catégories socio-professionnelles comme marqueurs des combinaisons sociales localisées}

Le découpage en sept catégories socio-professionnelles (CSP) proposé par les recensements de 1976 et 1986, combinaison de statuts dans l'emploi et de branches d'activités, permet d'approcher la distribution sociale dans la région du Caire. Ces catégories reprennent, en les regroupant de façon spécifique, la nomenclature du Bureau international du travail et se rapprochent de la manière anglo-saxonne ${ }^{14} \mathrm{de}$ dessiner les contours du monde du travail (cf. annexe). Elles livrent une image schématique, certes, de la société égyptienne, mais renvoyant néanmoins à la manière dont elle-même se représente.

13 Cette taxinomie ne peut être confondue avec une hiérarchie; elle n'est cela qu'en partie. Ainsi, la catégorie "gens du commerce» regroupe les commerçants à leur compte, les salariés, les journaliers et les aides familiales non rémunérées travaillant dans le commerce de détail ou de gros... Elle classe la population active de plus de 15 ans ayant déjà exercé un emploi, travaillant ou non, dans le secteur formel ou informel. Nous avons retenu pour nos comparaisons les sept catégories concernant les activités explicitement définies ${ }^{15}$.

14 Même à proximité du Caire, le socle des agriculteurs tient une place non négligeable et nous rappelle que le paysan, pivot identitaire d'une Égypte fortement dépendante de son étroite vallée fertile, s'active non loin de la capitale (13,4\% du total des actifs dans la région du Caire). Les ouvriers de l'industrie et des transports $(37,1 \%)$ forment la masse des Cairotes et la seconde composante de l'espace rural autour du Caire. Cette catégorie rassemblant le plus d'actifs nous montre à quel point Le Caire demeure le pôle industriel de l'Égypte. Ceci peut apparaître comme une évidence, mais il faut noter ici que certaines capitales du dit tiers monde agrègent des populations sans pour autant que s'y développe un socle productif tandis que, parallèlement, les grandes capitales des démocraties industrielles se caractérisent par le poids de plus en plus faible des ouvriers et par leur tertiairisation. Ce dernier processus est d'ailleurs en partie en cours au Caire, comme en témoigne la part croissante des cols blancs qualifiés, ingénieurs, techniciens et enseignants, sans que la part des ouvriers ait fléchi entre 1976 et 1986. Dans la mesure où l'ouverture aux capitaux étrangers et la création d'usines d'assemblage concernent surtout les villes nouvelles du Six-Octobre et du Dixde-Ramadan, la polarisation de l'activité industrielle au Caire ne semble pas fléchir. Seule la capitale et ses prolongements offrent les infrastructures et les facilités de communications internationales qui autorisent de nouveaux développements. En 1986, 
la région du Caire rassemblait $43 \%$ de la population ouvrière d'Égypte contre $45 \%$ en 1976.

15 Les employés de bureau fortement corrélés avec le secteur public forment le second pôle de l'Égypte urbaine et symbolisent encore l'encadrement des campagnes par les services de la République (12,4 \% du total des actifs dans la région du Caire). D'aucuns les désigneront volontiers comme une masse réduite à la passivité bureaucratique et qui, depuis les réformes nassériennes, paralyse les initiatives de développement. Ce sont encore les héritiers de cette figure emblématique, au cœur de tous les récits sur le peuple cairote, de l'effendi promu par l'école.

Les diplômés du supérieur, c'est-à-dire les ingénieurs et les techniciens du privé et du public,les enseignants et les médecins, composent le bataillon de la «modernité en marche", celui-là même qui devrait s'afficher comme l'emblème des efforts d'édification d'un appareil universitaire moteur d'une promotion sociale et économique globale et individuelle ( $18,3 \%$ du total des actifs dans la région du Caire). Noyau traditionnel de la ville arabe, les marchands s'exposent à travers un regroupement d'activités et de statuts, socialement peut-être encore plus hétérogène que les autres, où se mêlent marchands ambulants, marchands d'or, vendeurs de véhicules importés et employés, salariés ou non ( $7,8 \%$ du total des actifs). Parmi ces catégories, il en est une difficilement réductible en un mot. Elle regroupe, d'une part, les propriétaires et les actifs des services privés ainsi que la domesticité et, d'autre part, les employés des services publics intervenant sur la voirie (policiers, pompiers...). Dans toutes nos analyses, ce groupe des services se caractérise par sa volatilité géographique (9,4\% du total des actifs).

17 Enfin, le groupe des dirigeants du public et du privé rassembler l' " élite » évoquée par John Waterbury ${ }^{16}$ et forme $1,7 \%$ du total des actifs. Par ses manières d'agir, de se reproduire et d'échanger avec le système économique international en utilisant la citadelle bureaucratique en rempart contre la concurrence, elle entretient largement le fossé toujours plus marqué entre l'ostentation et la paupérisation.

\section{Des modalités socio-professionnelles qui polarisent des différences sociales, économiques et culturelles}

18 Pas plus que dans d'autres pays, ce type de catégorisation ne rend compte de la subtilité des regroupements sociaux, et encore moins de la fluidité des relations et des imbrications, au Caire ou dans d'autres villes d'Égypte.

19 Toutefois, elle donne à voir une structuration de l'espace, des regroupements et, des recompositions qui mérite d'être mise en évidence. En outre, même s'il ne s'agit que de co-occurrence spatiale, la localisation de telle ou telle catégorie est en relation étroite avec la présence ou non d'autres variables. En particulier la variable éducative, dont on sait ce qu'elle véhicule comme indications sur le capital social des populations, est corrélée de façon hiérarchique avec la distribution selon nos sept catégories d'emploi (voir tableau 2 p. suivante).

20 Autrement dit, cette répartition en sept classes auxquelles se rattachent nombre de variables plus ou moins indépendantes reste une valeur heuristique significative. Elle donne à voir une image forcément synthétique de la distribution sociale, mais néanmoins nécessaire car mettant sur la piste d'une structure cachée organisant 
l'espace urbain, d'un «ordre moral» pour reprendre l'expression de Robert Ezra Park $^{17}$.

21 Il est incontestable, même si cela peut paraître grossier, que la localisation résidentielle associée à la position des individus dans le processus de production donne à voir une mise en ordre. Pour le moins, elle rend compte d'une certaine segmentation du marché du logement qui compose des localisations contraintes et des processus de filtrage, lesquels, à leur tour, conditionnent la reproduction du système urbain. Et en définitive, la division sociale de l'espace expose les valeurs d'une société, les conditions de son expansion et la manière dont se gèrent ses contradictions, ou encore la façon dont évoluent les cadres structurants de la scène urbaine.

Bien entendu, comme ces catégories distribuent les populations en fonction de leur lieu de résidence, elles ne permettent pas d'analyser les localisations et les redistributions d'activités. Ce travail nous éclaire simplement sur les qualités sociales de l'étalement, de la croissance périphérique et des recompositions plus centrales. Il introduit à une réflexion sur la manière dont la hiérarchie sociale qui caractérise la totalité mégapolitaine se décline en espaces mixtes et en espaces spécialisés et désigne ainsi les filtres qui réduisent les chances d'une multiplication des interactions sociales.

Toutefois, à aucun moment l'on ne saurait retenir l'hypothèse d'une ségrégation. Nous savons en effet trop peu de choses sur les mobilités et les échanges, donc sur les interactions sociales grandement favorisées par la réduction des distances et le foisonnement des flux, par les proximités que composent les densités extrêmes d'une ville telle que Le Caire. Nous observerons simplement des mises à distance, en termes de résidence, qui renvoient à des choix, à des stratégies et à des contraintes, à de simples structures donc, qu'il serait abusif d'interpréter comme des enfermements.

Tableau 2 : Matrice de corrélation entre la distribution relative des modalités éducatives* et sociales en 1986 dans la région du Caire

\begin{tabular}{|l|l|l|l|l|l|l|l|l|}
\hline & Analphabète & Alphabétisé & $\begin{array}{l}\text { Primaire } \\
\text { acquis }\end{array}$ & $\begin{array}{l}\text { Préparatoire } \\
\text { non acquis }\end{array}$ & $\begin{array}{l}\text { Préparatoire } \\
\text { acquis }\end{array}$ & $\begin{array}{l}\text { préparatoire } \\
\text { universitaire }\end{array}$ & $\begin{array}{l}2^{\text {er }} \text { et } \\
3^{\mathrm{e}} \\
\text { cycle }\end{array}$ \\
\hline Agriculteurs & 0,78 & $-0,24$ & $-0,56$ & $-0,68$ & -0.72 & $-0,88$ & $-0,81$ & $-0,32$ \\
\hline Services & $-0,23$ & 0,2 & 0,17 & 0,13 & 0,18 & 0,03 & 0,16 & 025 \\
\hline $\begin{array}{l}\text { Gens du } \\
\text { commerce }\end{array}$ & $-0,34$ & 0,01 & 029 & 0,39 & 0,33 & 0.36 & 0,22 & 0,07 \\
\hline $\begin{array}{l}\text { Employés } \\
\text { de bureau }\end{array}$ & $-0,69$ & 0,15 & 0,42 & 0,68 & 0,70 & 0,71 & 0,44 & 0,15 \\
\hline \hline Ouvriers & $-0,05$ & $-0,34$ & 0,3 & 0,21 & -0.04 & 0,01 & $-0,25$ & $-0,29$ \\
\hline $\begin{array}{l}\text { Ingénieurs, } \\
\text { techniciens }\end{array}$ & $-0,88$ & $-0,13$ & 0,36 & 0,56 & 0,89 & 0,77 & 0.94 & 0,72 \\
\hline
\end{tabular}




\begin{tabular}{|l|l|l|l|l|l|l|l|l|}
\hline Dirigeants & $-0,68$ & $-0,04$ & 0,16 & 0.30 & 0,69 & 0,50 & 0,91 \\
\hline
\end{tabular}

* Niveau scolaire des personnes de plus de dix ans.

\section{Le Caire arrimé au système-monde}

La mise en évidence de la structure socio-spatiale cairote et son analyse ont déjà été abordées. En fait, dèsla décennie 60, Janet Abu Lughod a borné le terrain en s'appuyant sur les données censitaires de 1947 et $1960^{18}$. Il y eut même dès 1934, avec la thèse de Marcel Clerget sur Le Caire, une tentative pionnière de topographie sociale intraurbaine qui n'avait alors que peu d'équivalents, même pour les villes européennes ou nord-américaines ${ }^{19}$. Ce dernier se plaçait déjà dans la perspective d'une géographie sociale, malgré l'énoncé de jugements que l'on pourrait qualifier d'ethnocentrés.

A la lumière des changements intervenus depuis 1947, Janet Abu Lughod a pu proposer un schéma raisonné du Caire en 1960. Il s'y déploie très clairement un ordre social en devenir. Ensuite, au fur et à mesure qu'elle affinait son analyse et qu'elle suivait l'affirmation des habitants du Caire dans leur position de citadin, elle dégageait de plus en plus nettement une condition urbaine se faisant l'écho des réflexions de Louis Wirth ${ }^{20}$ quant au " phénomène urbain comme mode de vie » (1938) et des débats qui se prolongent jusqu'à présent sur ce sujet. De même, dès 1961, elle avait très largement remis en cause le thème de la «villagisation » du Caire ${ }^{21}$. Il a pourtant continué à hanter les recherches urbaines malgré tout ce que l'on a pu apprendre, depuis les années soixante, sur le fléchissement très net des flux migratoires vers la capitale et la domination des migrations ville-ville en partie polarisées par Le Caire. Entre 1976 et 1986 , le solde migratoire en faveur de la capitale n'a contribué que pour $10 \%$ à sa croissance.

26 A des degrés divers, dans un contexte d'urbanisation diffuse irradiant jusqu'aux plus petits bourgs ruraux, la condition urbaine touche à présent l'ensemble de la scène égyptienne ${ }^{22}$. Selon un jeu inégal, la tradition rurale et les relations de face à face et communautaires qui la caractérisent sont déstabilisées par la multiplication des échanges et des rapprochements avec le système des villes. A l'immédiateté se substitue la médiation. Il suffit, pour le comprendre, de dire qu'en 1986,36 \% des actifs ruraux - soit $10 \%$ de plus qu'en 1976 - étaient engagés dans des activités non agricoles sur place, dans des bourgs proches ou dans des villes (voir graphique 1 page suivante).

L'Égypte s'inscrit donc bien dans un mouvement universel dont, dès 1857 dans les Grundisse, Karl Marx ${ }^{23}$ soulignait l'importance: "L'histoire moderne, c'est celle de l'urbanisation de la campagne et non, comme dans l'Antiquité, celle de la ruralisation de la cité. » Paradoxalement, la résistance de cette représentation en termes de ruralisation peut précisément se comprendre à la lumière de ces mêmes écrits de Karl Marx ou encore de l'essai sur la ville de Max Weber (1921). Tous deux ont en effet défini la ville orientale comme une catégorie à part, dominée par la campagne et dont les principes associatifs seraient faibles. L'absence d'autonomie de la communauté urbaine, dans un contexte de centralisme faisant référence au " despotisme oriental », est aussi invoquée ${ }^{24}$. Nous sommes donc bien sur une scène urbaine en devenir où des 
familles que l'on peut rattacher à des groupes sociaux cherchent leur place, se déplacent et produisent de la territorialité.

Graphique 1 : Distribution des actifs ruraux en 1986

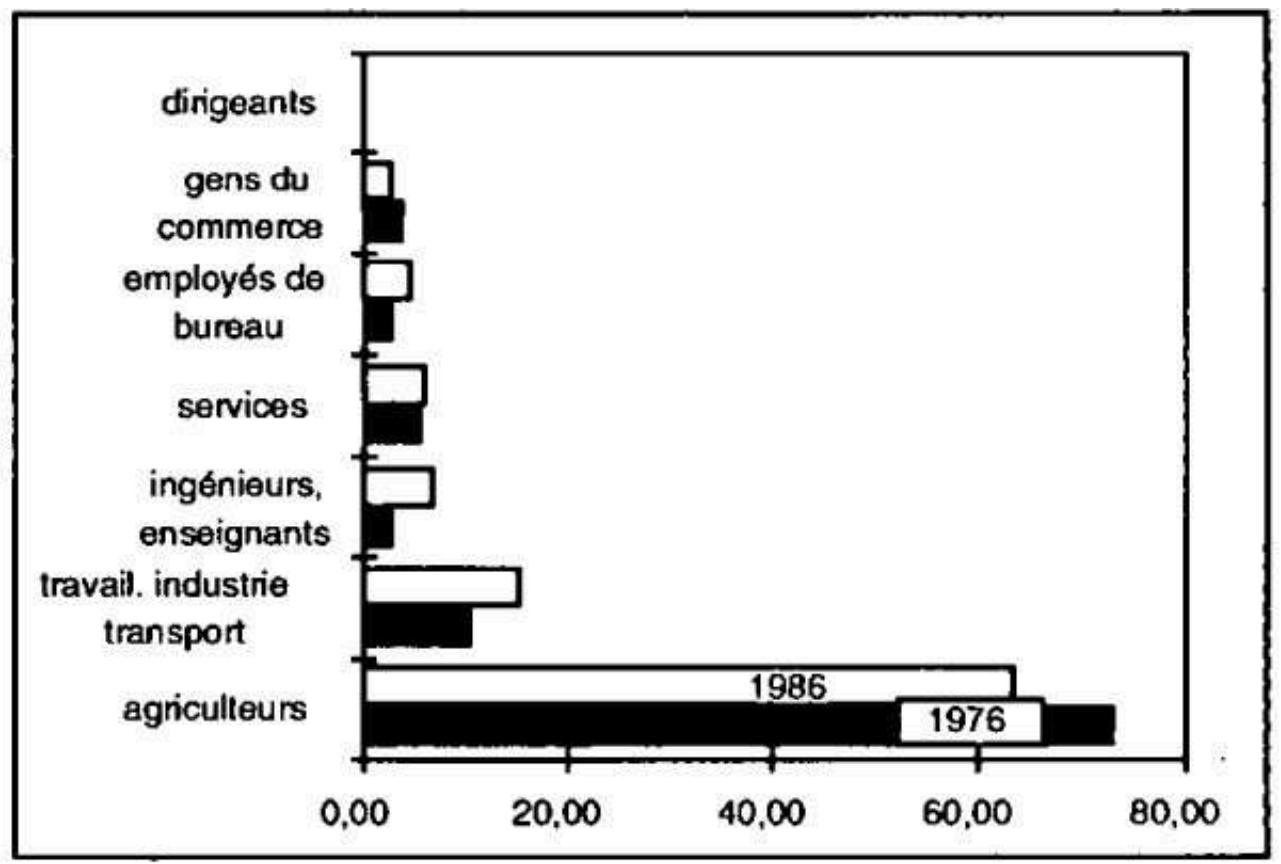

Enfin, en 1990, Janet Abu Lughod - dans une comparaison entre New York etLe Caire où elle conclut, dans le contexte de l'unification du système monde, à l'émergence de villesmonde intégrant des dimensions de villes du tiers-monde et inversement - , achève d'associer Le Caire au devenir de la division internationale du travail. Par là même, elle souligne toute la pertinence d'analyses qui chercheraient à tester l'homothétie des bouleversements intra-urbains à travers le monde des villes. Elle-même a suivi les dynamiques sociales intra-urbaines dans cette transition globale de la ville préindustrielle à la ville industrielle, selon sa propre terminologie ${ }^{25}$. Elle nous propose de la suivre plus loin encore en rappelant qu'il n'y a pas de rupture mais un ajustement progressif entre les héritages et la modernité culturelle, industrielle, commerciale et politique transnationale, ce qu'elle appelle « le paradoxe de la nature inchangée face au changement permanent». Le Caire et l'Égypte des villes en général ne sont pas réductibles à des figures stéréotypées supposées rendre compte de l'urbanisation du tiers monde ou du monde arabe. Le Caire peut tout aussi bien être rapproché de New York mais encore, à l'échelle régionale, de l'urbanisation diffuse de Java ${ }^{26}$. L'ajustement local des contraintes universelles inhérentes au monde des villes dessine la personnalité du Caire.

Mais il ne faudrait pas, selon un raccourci courant relevant de la mécanique plus que de la logique formelle, comprendre cette universalité ajustée comme le résultat d'une globalisation. A la lumière de méthodes universelles apparaissent des dynamiques urbaines comparables qui renvoient à la cohérence du système des villes et à des contraintes matérielles incontournables qu'en tous points il rencontre. La dynamique des villes était comparable avant qu'il soit question de mondialisation. A travers une analyse précise de la redistribution des catégories socio-professionnelles entre 1976 et 
1986, nous serons en mesure de préciser ce qu'implique l'affirmation du Caire comme ville-relais intégrée au système monde.

\section{De l'émergence de l'informalitéà l'illégalité généralisée}

Depuis le milieu des années quatre-vingt, Galila el Kadi a développé une analyse de la division sociale de l'espace cairote mettant en lumière une forme nouvelle caractéristique de l'urbanisation des années soixante-dix/quatre-vingt-dix, à savoir l'habitat « informel » ou « spontané », pour ne retenir que les deux expressions les plus utilisées. La forte représentativité des couches sociales à bas revenu dans les zones périphériques de formation récente ne leur confère pas, cependant, une homogénéité sociale interne. Des disparités sociales existent entre les secteurs les composant, et même à l'intérieur de chaque secteur ${ }^{27}$.

31 Malgré ces travaux invoquant la prudence, le sens commun a posé une chape d'uniformité sur cet espace périphérique en devenir. La représentation des nouveaux quartiers non planifiés a été noyée dans la catégorie de l'» informel », voire du " slum » ou encore $d u$ " hasardeux » (selon une traduction possible du mot arabe 'ashwaiyyât, employé pour désigner ces quartiers). Aujourd'hui ce ne sont pas moins de $50 \%$ des Cairotes qui résident dans ces zones non planifiées, loties en défiant la légalité, sans permis de construire, parfois sans propriété du terrain comme sur les zones publiques squattées. Dans ce fourre-tout sont amalgamés les foyers actifs de l'habitat sousstandard qui, en bien des endroits, renferment de puissantes capacités de promotion, et les réceptacles précaires de mobilités très fortement contraintes où la survie s'envisage au jour le jour... Toutes les nuances sont possibles.

Les 'ashwaiyyât sont néanmoins devenues une catégorie "naturelle", massive, compacte, impénétrable pour tout dire, qui en est même arrivée à désigner la totalité de l'espace urbain : « 84 \% des immeubles bâtis au Caire, à Giza et à Shubra al-Khayma sont 'ashwaiyyât. $16 \%$ des constructions disposent d'un permis. ${ }^{28}$ Ce n'est donc plus une catégorie qui permet de qualifier des quartiers, mais un système largement répandu.

En outre, la catégorie 'ashwaiyyât a de plus en plus une fonction sociopolitique : elle est désignée comme le lieu de toutes les menaces, dela contestation islamiste comme de la violence sociale. Il est donc important, voire nécessaire, que ces 'ashwaiyyât apparaissent comme une masse obscure, globalement négative. Ainsi l'édifice social et politique n'est-il pas structurellement remis en cause. Il existe un « ennemi » localisé. Ce marquage pourrait jouer en laveur d'une dualisation et d'une réaction massive et violente des populations ainsi désignées, mais ce serait sans compter précisément sur la pluralité et les hiérarchies socio-spatiales internes de cet espace amalgamé. Dans les faits, la distinction l'emporte sur la solidarité, cette dernière demeurant effective simplement à l'échelle de la rue, et encore, pour quelques questions d'intérêt général ${ }^{29}$. L'évidence intuitive de la catégorie même de 'ashwaiyyât génère une ambiance globale spécifique, comme une nouvelle identité. Une ambiance tellement prégnante que toute la ville s'y perdrait peu à peu, composant « une image unitaire. Unité dans la laideur? », pour reprendre l'interrogation de Mercedes Volait, ou encore, sombrant « dans un vaste continuum spontané s'étendant du centre à la périphérie », selon Galila el Kadi ${ }^{30}$. 
Non seulement l'informalité "grignote» les terres agricoles, mais elle «ronge» l'intérieur de la ville, la « gangrenant »...

L'idée de hasard contenu dans le terme 'ashwaiyyât permet peut-être de comprendre ce que cela voudrait désigner. La dynamique urbaine actuelle se vit et se donne à voir largement de cette façon, contrainte par le hasard ou la fatalité, ou plutôt par les miracles de la main invisible et de ses acteurs, des hommes d'affaires guère plus apparents. En outre, le secteur de la construction, canal d'expression privilégié de la libre entreprise en Égypte, se trouve particulièrement à son affaire dans les quartiers dits informels. D'aucuns ont donc intérêt à ce que ces développements restent précisément 'ashwaiyyât, c'est-à-dire à ce que l'État absent ne puisse pas imposer des normes coûteuses.

$\mathrm{Au}$ moment où ce dernier, lancé dans une vaste politique d'ajustement structurel, ne cesse d'ouvrir le champ des possibles, les périphéries en construction peuvent d'autant plus aisément rester un vaste domaine de spéculation immobilière ignoré, et ces activités peuvent même s'étendre vers le centre sans rencontrer d'obstacle majeur.

La qualité sous-standard du bâti n'empêche pas sa commercialisation. Au contraire, elle la rend accessible à davantage de familles. De plus, le caractère informel d'un quartier ne l'empêche pas d'être connecté à divers réseaux, à l'eau, à l'électricité et plus tard aux égouts. Avec les mesures d'ajustement, les réseaux publics ne sont plus un simple instrument politique. Il n'est plus aussi simple, pour les services de l'État, d'interdire la desserte et l'équipement d'un quartier sous prétexte qu'il a été construit illégalement. Pour rentabiliser les amenées, les services compétents raccordent les nouveaux immeubles dès que les familles et les entreprises sont en mesure de payer. Avec des campagnes d'installation de compteurs, cette rationalisation économique de la politique de raccordement se traduit aussi par une réduction du nombre de branchements illégaux. En conséquence, un nombre croissant de familles, ne pouvant payer des factures en constante augmentation, se trouvent privées de services élémentaires comme l'eau. Ces hausses visent à compenser la réduction des subventions publiques qui permettaient de maintenir une offre ne couvrant pas les coûts de production et de desserte. Devenir bénéficiaire des réseaux publics, premier pas vers le statut de citadin, est devenu à la fois plus aisé et plus sélectif, ce qui contribue par là même à brouiller les limites socio-spatiales. Désormais, ces dernières suivent davantage une logique réticulaire, alors que les politiques massives de raccordement ou le refus de raccorder la totalité d'une zone tendent à disparaître.

En adhérant à la catégorisation désignée par le terme 'ashwaiyyât, nous sommes renvoyés à l'impossibilité de caractériser les mégapoles. Le Caire rejoint celles qui, "loin de structurer leur territoire, le 'bouffe' littéralement sans logique apparente ${ }^{31}$. Nous décrivons la ville sur la base d'une catégorie manipulée, instrumentalisée, et de toute façon trop englobante. Si nous ne nous en gardons pas, elle pourrait jouer un rôle sensiblement comparable à celui tenu en son temps par l'idée de ville "arabe", « islamique» ou "orientale $»^{32}$. L'informel enfermerait l'urbanisation et les recompositions sociales qu'elle entraîne. Autrement dit, Le Caire quitterait sa dimension "orientale" pour faire naufrage dans le «hasardeux", cette nouvelle «barbarie ».

Cette catégorie ne peut donc qu'être contournée. Suivant le dessein qui est le nôtre, Le Caire doit rester une mégapole traversée par des recompositions socio-spatiales à travers lesquelles, comme ailleurs, se dessinent des territoires. Et cela, même si ces 
derniers sont le reflet d'une mise en ordre qui s'appuie sur la logique des 'ashwaiyyât, dont la prégnance est indéniable, mais sans doute insupportable à vivre au quotidien. Certes, la condition urbaine est très altérée par les contraintes de l'informalité, mais derrière cet écran n'en subsistent pas moins un espace social et une lutte pour l'accès à la ville.

Carte $\mathrm{n}^{\circ} 1$ : La région du Caire

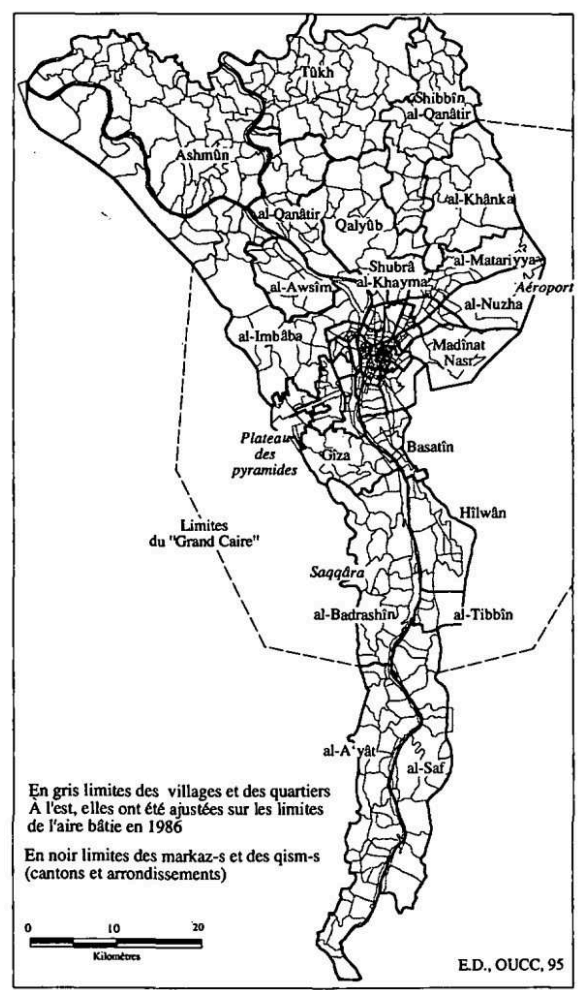


Carte $n^{\circ} 2$ : L'agglomération du Caire

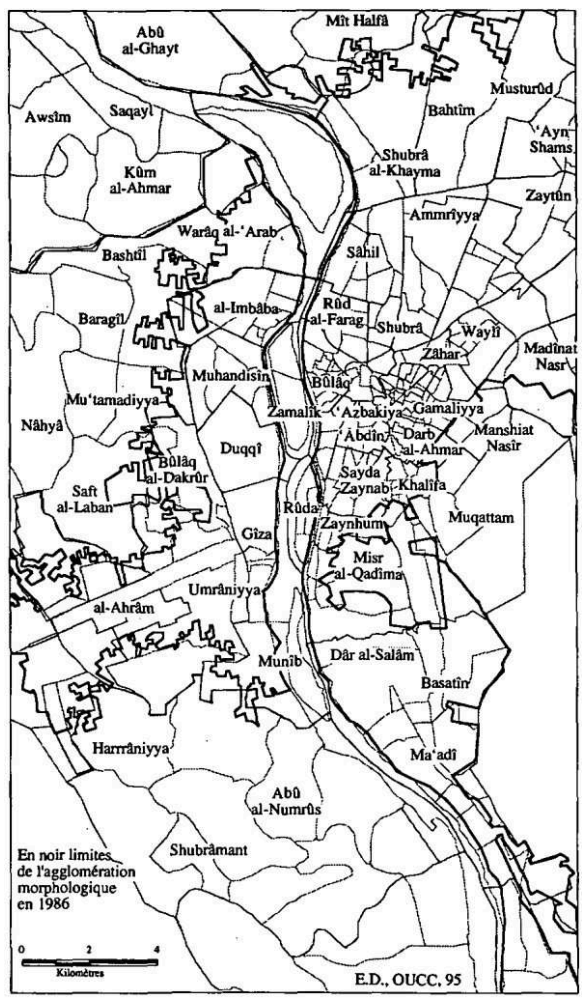

\section{Redistributionset étalement}

\section{Des densités recomposées}

Alors que l'espace urbain s'élargit, déborde sur la vallée fertile et ses franges désertiques, s'opère un glissement d'une certaine mixité sociale centrée vers d'autres combinaisons et d'autres distances sociales. En particulier, des zones de plus en plus spécialisées sont susceptibles de s'affirmer. S'habillant en mégapole, la métropole ne s'écarterait-elle pas d'un des fondements de l'urbanité, à savoir de la mixité ou du regroupement dans des métriques de proximité de populations hétérogènes? Mais cette spécialisation de l'espace est-elle si généralisée ? La question reste posée.

Il nous paraît pertinent de focaliser ici notre attention sur cette échelle intermédiaire où se dessine la totalité à traversla nuance des parties, c'est-à-dire sur une région du Caire volontairement très large, pour être sûr de couvrir l'ensemble de l'aire concernée par les puissantes dynamiques sociales de la capitale égyptienne. Cette aire s'étend au nord jusqu'à Tûkh et Ashmûn,et au sud, jusqu'à al-Saf et al-A'yât. Elle est donc plus étendue que la région administrative dite du « Grand Caire ». Découpée en 620 qura et shiyakha (villages et quartiers), soit, en moyenne, en unités de 20.000 habitants, mais avec des minimums autour de 1.500 et un maximum dépassant 280.000 habitants, notre région du Caire élargie comptait quelque 12 millions d'habitants en 1986 (voir cartes 1 et 2).

Les pertes d'habitants dans les quartiers centraux et péricentraux aux densités extrêmes - ayant atteint un seuil de saturation - sont corollaires de ce processus d'étalement. La réduction du nombre de shiyakha comptant plus de 1.000 habitants par 
hectare (66 en 1976,40 en 1986) en témoigne, de même que la plus faible densité moyenne des unités urbaines (500 habitants par hectare en 1986, contre 564 en 1976). Ce mouvement est le produit complexe de reconquêtes foncières, de la dégradation du cadre bâti comme des stratégies familiales d'accès à la propriété en périphérie, ou de décohabitations qui touchent à la fois d'anciens faubourgs populaires comme Bûlâq et Shûbra, et des quartiers encore plus centraux et leurs prolongements sudet nord. Il englobe des quartiers comme Zaynhum Sayyida Zaynab, Khalîfa, Darb al-Ahmâr, alMuski ou Gamâliyya. Toutefois, ces derniers dessinent toujours l'axe historique des fortes densités qui s'étire de part et d'autre des avenues Bûr Sa'îd et al-Gaysh, c'est-àdire le long de l'ancien Khalîg, avec un élargissement à l'est buttant sur l'enfilade des cimetières qui, à l'image d'une colonne vertébrale, a structuré l'expansion sud-nord de la fondation de Fûstat, à la fin de l'époque mamelouke.

Bien entendu, cette dédensification correspond à ce l'on sait sur l'étalement continu du cadre urbanisé. Il ne fléchit pas, même dans les métropoles dont la population s'est stabilisée. Au Caire, nous sommes encore loin de cette situation. L'étalement est à peine un desserrement mais plutôt un mouvement puissant, parfois même brutal, de densification des quartiers périphériques en l'absence de terre agricole de valeur, comme à Matariyya (+ 4,5\%/an entre 1976 et 1986), 'Ayn Shayms (+ 7,8\%/an) ou Basatîn (+ $9 \% /$ an). Sur les terres agricoles, comme à Imbâba, il rejoint des valeurs extrêmes (1.000 à 1300 hab/ha) autour desquelles la densité se stabilise (cf. carte 3). Plutôt que d'étalement,ilfaudrait parier d'un débordement minimum marquant particulièrement l'arc nord de l'agglomération. En outre, du strict point de vue de l'agglomération morphologique, c'est-à-dire de l'extension du cadre bâti, Le Caire ne s'est étendu, de 1947 à 1989, que de $80 \mathrm{~km}^{2}$ pour atteindre $340 \mathrm{~km}^{2}$ quand, dans le même temps, sa population quadruplait.

L'urbanisation sur la trame agricole obéit à une rationalitési forte qu'elle a produit très rapidement, en moins de vingt ans, des densités comparables à celles du tissu central ancien, dont la trame initiale a près de mille ans. La ville s'impose immédiatement, et le coût du foncier ne suffît pas à l'expliquer. Rien à voir, donc, avec une logique de banlieue, plus présente en revanche sur les trames désertiques plus ou moins planifiées de Madînat Nasr ou Nuzha, L'extension du Caire n'est que partiellement le produit d'une mise en ordre socio-fonctionnelle, d'un zoning qui distingue les occupations du sol, les dissocie. Activités et populations demeurent largement mêlées. Autrement dit, la dynamique centrifuge qui anime le Caire opère à la fois une dissociation des populations antérieurement plus proches, même si la distance sociale s'imposait à travers des micro-divisions de l'espace dans une ville très compacte, coincée entre le Nil et le Muqattam, et une mixité plus grande de périphéries auparavant polarisées par l'activité agricole. 


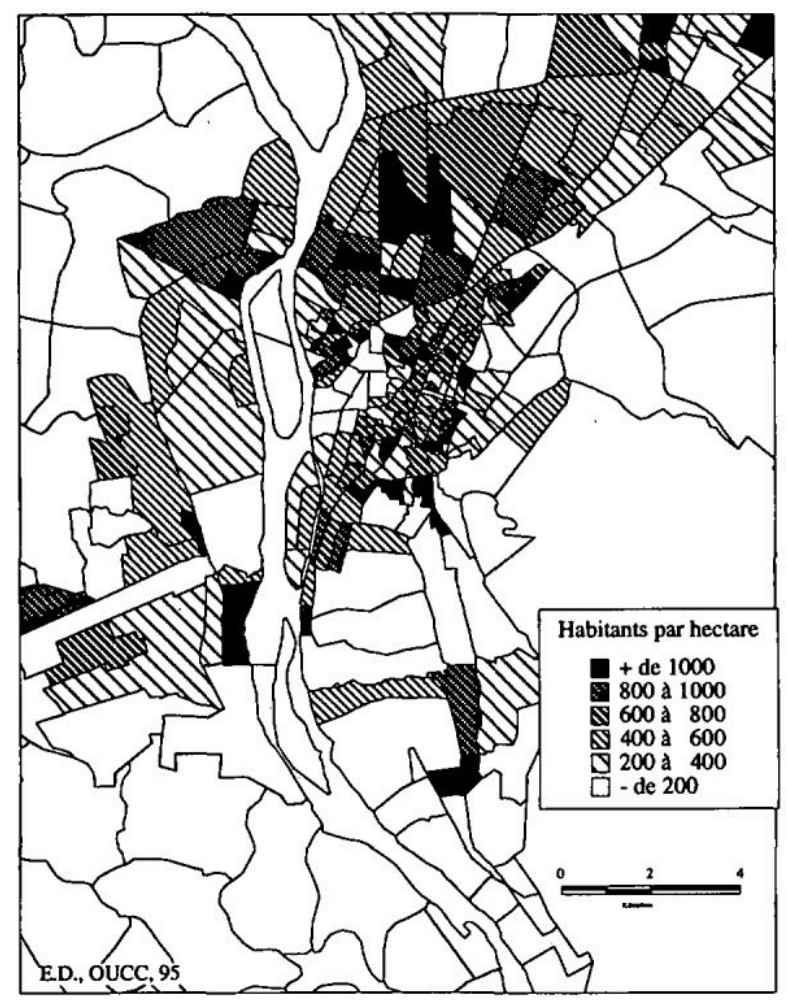

Si nous retenons comme espace central un territoire borné au nord par les limites extérieures des quartiers de Bûlaq et de Shûbra, au sud par Misr al-Qadîma, débordant à peine sur la rive ouest du Nil de 'Aguza à Doqqi, buttant à l'est sur le Muqattam et excluant Heliopolis, nous obtenons une aire d'à peine $75 \mathrm{~km}^{2}$, qui compte pour moins de $10 \%$ de l'agglomération actuelle.Ce noyau central, composé des territoires (20 qism où arrondissements) où s'est constitué, Le Caire jusqu'à la fin du XIXe siècle, regroupait encore près de la moitié de ses habitants en 1976, mais déjà plus qu'un tiers en 1986. Ce changement de rapport rend compte du poids croissant des auréoles successives qui forment une ceinture - certes incomplète et, du point de vue social, fortement sectorisée - autour d'une centralité dont la masse se réduit en valeur relative, voire en partie en valeur absolue. Les 16 qism les plus centraux ont en effet perdu, de 1976 à 1986 , quelque 412.000 habitants. Néanmoins, en 1986, ils concentraient toujours $23 \%$ de la population du Caire, sur à peine $6 \%$ de sa surface ${ }^{33}$. Nul doute que cette zone d'habitat ancien, ravaudée de nouvelles constructions et de surélévations aux assises incertaines, a dû voir sa population baisser encore depuis le tremblement de terre d'octobre $1992^{34}$.

\section{Jusqu'où va la ville?}

Pour comprendre ce que recouvrent les redistributions sociales qui, entre 1976 et 1986, ont animé Le Caire, encore faut-il savoir jusqu'où s'étend la mégapole. Sinon, il est impossible de réfléchir en termes de redistribution. Autrement dit, la question pourrait être : jusqu'où interfère le mode de vie urbain? Nous ne pouvons nous satisfaire des limites administratives car, dans ce cadre de gestion des équipements publics et de la police, le poids de l'urbain est très fortement sous-estimé. Par exemple, Warâq al-'Arab, qui comptait 127.000 habitants en 1986 , est, tant du point de vue morphologique que 
sociologique ou fonctionnel, un quartier de l'agglomération du Caire (nord-est de Gîza, Imbâba). Pourtant, il a conservé le statut de village jusqu'en 1993.

En première approche, nous avons pris un espace très étendu, c'est-à-dire une région du Caire qui rassemblait 12 millions d'habitants en 1986, sur $2.300 \mathrm{~km}^{2}$. Cette aire que nous avons, à dessein, très largement délimitée, permet de ne pas exclure a priori des dynamiques sociales qui ont à voir avec l'essor de la capitale égyptienne. Elle comptait à l'époque une part importante d'actifs agriculteurs, $13 \%$ en moyenne, répartis en amont et en aval de l'agglomération, avec des maximums pouvant atteindre $90 \%$. Considérant le poids des agriculteurs dans la population active comme un bon indicateur de ruralité - sans pour autant oublier que l'espace rural n'est pas qu'agriculture - , nous pouvons observer où se trouvent les limites sociales de l'espace mégapolitain. Ainsi, prenant un seuil maximum de 10\% d'agriculteurs, nous obtenons une délimitation de l'espace urbain tout à fait lisible dans les paysages. Elle correspond au front d'urbanisation et au seuil de l'aire comptant plus de 200 habitants par hectare.

Carte $n^{\circ} 4$ : Poids des agriculteurs en 1976

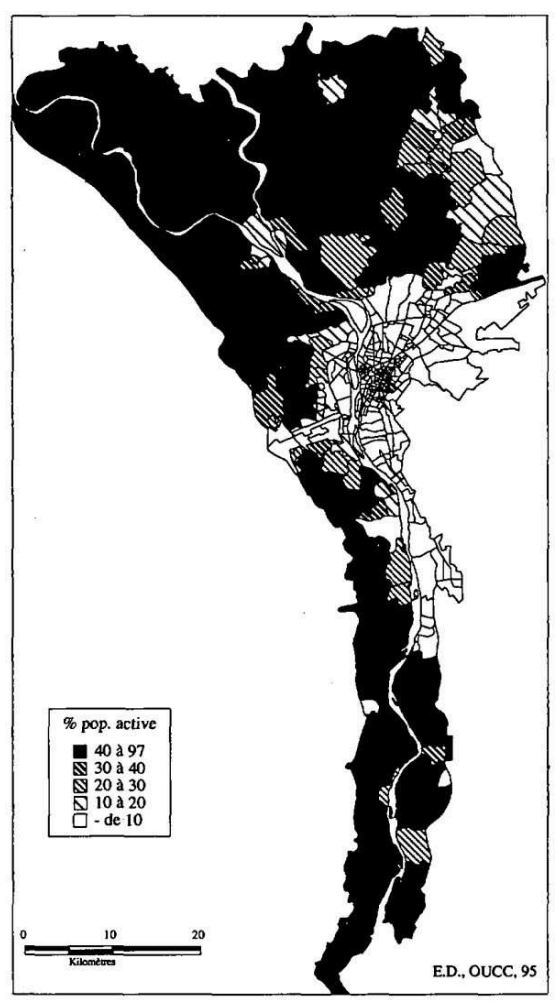




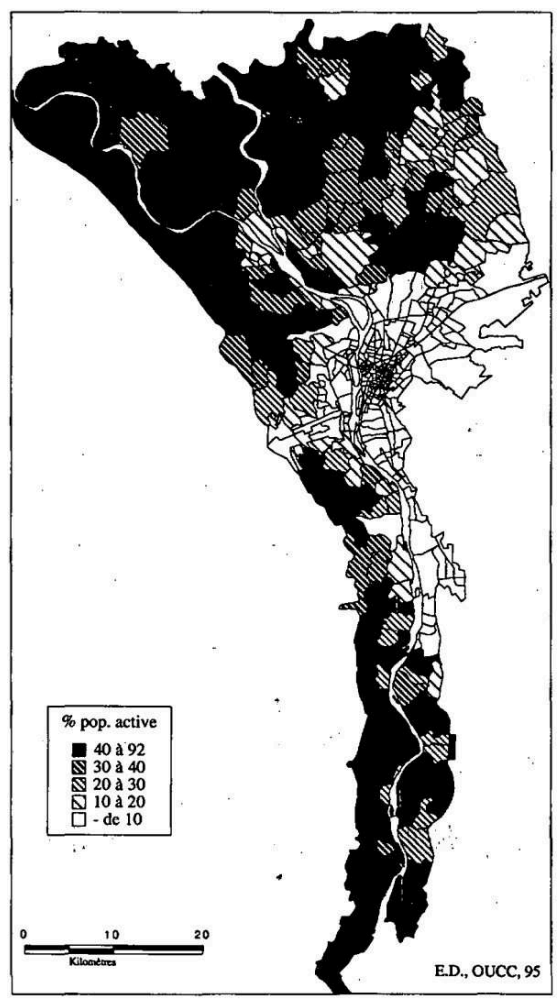

Entre 1976 et 1986, si la zone agglomérée des unités comptant moins de $10 \%$ d'agriculteurs, c'esl-à-dire à peine $5 \%$ en moyenne, est demeurée stable avec 295 shiyakha et qura, la composition sociale des unités plus périphériques s'altérait considérablement, alors que leur densité augmentait (voir cartes 4 et 5). Autour de ce noyau des moins de $10 \%$, le nombre d'unités comptant entre 10 et $20 \%$ d'agriculteurs est passé de 14 à 41. Au-delà de l'aire urbaine agglomérée en s'appuyant sur des villages, la dynamique urbaine s'est donc déversée dans des espaces encore très marqués par le travail des champs. Les modes de vie y ont été définitivement bouleversés par les activités productives urbaines et les changements dans les pratiques qu'elles imposent, notamment en ce qui concerne les mobilités et les modes de consommation. Le potentiel d'interactions se multipliant, le champ des possibles s'est ouvert, composant une mosaïque de nuances, de micro-combinaisons, que l'indigence générale des revenus ne parvient pas à unifier. Cet espace fortement recomposé regroupait 827.000 habitants en 1986, sur quelque 18.000 hectares. Entre 1976 et 1986, les lieux forts de la transition urbaine ainsi dégagés pourraient avoir fait définitivement passer de la campagne à la ville quelque 800 hectares par an. Toutefois, une large partie des surfaces communales continuent d'y être cultivées ${ }^{35}$.

Depuis 1986, nombre de ces unités, comme Bashtil, Barâgîl ou Saft al-Laban, pour ne désigner que les marges d'Imbâba et de Bûlâq al-Dakrûr au nord-ouest, sont venues s'agréger à la mégapole. C'est pourquoi, ensuite, il faudra entendre par « agglomération du Caire " l'ensemble continu des 333 unités qui comptaient moins de $20 \%$ d'agriculteurs en 1986. C'est-à-dire une conurbation comptant en moyenne 5,9\% d'agriculteurs. Cette limite supérieure se devait d'être souple afin d'intégrer les qura qui ont vu leur part d'agriculteurs chuter brutalement et dont on connaît aujourd'hui le degré d'insertion urbaine : par exemple, Barâgîl est passé de $51,4 \%$ à 24.8 \%, Saft al- 
Laban de 43,6 \% à 22,6 \% ou Qalag (al-Khânka) de 38,6 \% à 21,3 \%. Parmi les plus fortes chutes, signalons encore Sabîl (Imbâba), de 39 \% à 16,6 \%, ou Kunayyisa (Bûlâq alDakrur), de $38.6 \%$ à $11.6 \%$.

Ces marqueurs saisissent une part importante du changement social majeur des années quatre-vingt dans la région du Caire, à savoir la plus grande mixité de l'espace périmégapolitain en raison, surtout, du mouvement centrifuge qui a affecté une part non négligeable des Cairotes. En effet. $54 \%$ des personnes ayant effectué au moins une migration depuis leur naissance entre les trois gouvernorats composant la région du Caire (Gîza, Qaliubiyya, Le Caire) avaient quitté la capitale pour aller dans les deux autres gouvernorats.Cela concernait 510.000 migrants survivants saisis dans leur résidence périphérique lors du recensement de la population réalisé en 1986.

Cet élan centrifuge, ce débordement, nous indique que s'il s'est joué une amplification de la mixité sociale au Caire, c'est tout d'abord entre Le Caire et ses bordures rurales qu'il faut la voir, et au détriment des communautés caractérisées par la présence centrale des agriculteurs. Autrement dit, la mégapole a agi hors ses murs, donnant vie à de nouvelles formes de peuplement polarisées par des activités urbaines exercées en partie sur place ou engageant davantage de migrations circulaires. Ainsi a pris corps un continuum rural-urbain, les limites se sont altérées, la condition urbaine s'est imposée. Rien de plus banal que cette entropie du système urbain, même si elle revêt, ici comme ailleurs, une foule de figures particulières, notamment des villages urbains comptant plus de 40.000 habitants et des densités périphériques parfois supérieures à celles des quartiers centraux.

\section{Des distributions contrastées}

52 L'indicateur de base, pour rendre compte de la disparité d'une distribution dans un espace décomposé en sous-ensembles, est communément appelé indice de concentration. Employé pour décrire la manière dont les groupes sociaux occupent l'espace, se regroupent ou se mélangent, il est plutôt nommé indice de ségrégation ${ }^{36}$.

Tableau 3 : Indice de concentration

\begin{tabular}{|l|l|l|}
\hline Secteurs professionnels & 1976 & 1986 \\
\hline Ingénieurs, techniciens & 0,38 & 0,34 \\
\hline Dirigeants & 0,47 & 0,49 \\
\hline Employés de bureau & 0,24 & 0.18 \\
\hline Gens du commerce & 0,20 & 0,18 \\
\hline Services & 0,15 & 0,12 \\
\hline Agriculteurs & 0,81 & 0,59 \\
\hline Ouvriers & 0,30 & 0,28 \\
\hline
\end{tabular}


53 L'indice de concentration des agriculteurs a singulièrementchuté, passant d'un niveau indiquant une très faible représentation des autres catégories à une recomposition moins spécialisée engageant une diffusion des autres catégories. 1 signifie l'exclusion de toute autre catégorie, et 0 la mixité parfaite : 1 implique que dans les unités où la catégorie ainsi qualifiée est présente, elle en exclut totalement les autres.

La tendance au regroupement par catégorie a globalement fléchi en raison de l'urbanisation périurbaine. L'espace a gagné en mixité du fait de sa dilatation. A l'exception des «dirigeants » qui sont restés en marge de ces déplacements, les autres catégories ont toutes vu s'altérer, entre 1976 et 1986, leur manière de se distinguer par leur position dans l'espace. Il apparaît clairement que tous n'ont pas participé de manière uniforme à ce mouvement. A l'inverse, même, les dirigeants ont renforcé leur position au cœur de la cité, affirmant une logique de distinction.

55 Cette transformation vaut particulièrement pour les unités où le pourcentage d'agriculteurs est tombé au-dessous de $20 \%$. Elle est auréolaire, mais au-delà, elle devient réticulaire. Elle est structurée par les grands axes de part et d'autre du Nil, route de la Haute-Égypte sur la rive ouest, route agricole d'Alexandrie à travers le Delta, et relayée par des pôles secondaires comme Qanâtir, Qalyûb ou Badrashîn.

56 Elle est encore plus marquée, en termes d'étendue, à Qalyûbiyya. S'appuyant sur les franges est, c'est-à-dire sur l'ancien axe du canal d'Isma'îliyya, elle peut rejoindre le district industriel de al-Khânka/Abû Za'bal. Ainsi, progressivement, se dessine un couloir fonctionnel intégré depuis le terminus nord du métro à al-Marg. Là, il suffit de changer de quai - et d'attendre - pour trouver un train desservant toutes les gares jusqu'à Shibîn al-Qanâtir en bordure du désert oriental. Les terres les plus fertiles résistent davantage à l'urbanisation ${ }^{37}$. Ce développement péri-désertique est favorisé par l'urbanisation planifiée des marges désertiques, par l'essor des sites d'al-'Ubur et, plus loin, de Dix-de-Ramadan.

57 A l'ouest, les conditions de l'accrochage avec le désert sont loin d'être réunies. De plus en plus saturées, al-Ahrâm et Faysâl, les deux seules artères qui permettent d'accéder au désert et à la route d'Alexandrie, ne sont pas en mesure d'assurer le débordement de l'urbanisation sur les franges désertiques. De même qu'à l'est, l'étalement a été contrarié par les hauteurs du Muqattam, le plateau des Pyramides de Gîza à Kardasa, classé patrimoine mondial, impose un décalage vers l'aval. Le blocage du périphérique à cette hauteur et la mobilisation de l'Unesco le montrent bien. Tant que les axes de Bûlàq al-Dakrûr, via Saft al-Laban-Nahya et d'Imbâba via Barâgîl, ne seront pas complétés et connectés à la route circulaire et au désert, donc à la ville nouvelle du SixOctobre, les terres agricoles; de part et d'autre de ces couloirs s'urbaniseront. L'arrimage au désertest incontournable et il suppose encore une période d'étalement vers l'ouest de la pointe du Delta sur une étendue d'environ $25 \mathrm{~km}^{2}$. Même si le tracé restrictif de la route circulaire prétendait l'interdire et contenir I'agglomération du Caire comme l'unedes ville les plus denses du monde, l'étalement semble pouvoir être; satisfait par ces axes qui ont réussi à déborder ce qui, à la fois, se voulait un mur et une voie de circulation. Ce boulevard périphérique avait en quelque sorte l'ambition de clore une ville saturée et, par là meme, de contribuer à son asphyxie, alors que le parc d'automobiles privées ne cesse de s'accroître. 


\section{Redistributions et étalement}

Un second indice souvent utilisé, l'indice de similitude, permet de rendre compte de la proximité ou de la distance entre les groupes sociaux comparés deux à deux, en termes $\mathrm{d}^{\prime}$ «association » et de « rejet ». Il varie aussi de 0 à 1, 0 indiquant que la distribution relative de la catégorie $\mathrm{X}^{1}$ est comparable à celle de la catégorie $\mathrm{X}^{2}$ et 1 qu'elle est radicalement différente.

En 1976 comme en 1986, dirigeants et agriculteurs composaient des polarités très répulsives, plus encore que celles qui opposaient dirigeants et ouvriers. Les dirigeants ne cohabitaient qu'avecles actifs avec lesquels ils pouvaient partager quelques valeurs, à savoir les ingénieurset les employés de bureau, composant ainsi, l'identité des quartiers chics. La convergence ou l'ancrage plus affirmé, dans les mêmes espaces, des ingénieurs et des dirigeants s'impose. Toutefois, elle ne concerne pas tous les ingénieurs, techniciens et enseignants puisque, dans le même temps, la dissimilitude de ces derniers avec les ouvriers décroît sensiblement. Or,si l'on ajoute que les ouvriers demeurent très éloignés des dirigeants, cela nous met sur la voie d'une bipolarisation de la catégorie intermédiaire des ingénieurs. Une partie d'entre eux, fragilisés ou n'ayant jamais pu s'insérer véritablement au cœur de la ville, est engagée dans le mouvement de conurbation des périphéries (voir tableau 4).

S0 Le troisième pôle autour duquel s'agence l'espace cairote et ses environs peut se lire comme celui où se mêlent les actifs des services,les commerçants, les employés de, bureau et les ouvriers. On peut y reconnaitre les quartiers populaires du centre qui se caractérisent par une résistance de la diversité. A cette échelle, ces catégories sont celles autour desquelles s'organise un espace mixte ou subdivisé selon des marquages indépendants de l'appartenance sociale, au sens des catégories dont nous disposons. Cette composition mixte se maintient, voire se renforce, entre 1976 et 1986.

61 Davantage impliqués dans le processus de péri-urbanisation que les autres catégories, les ouvriers voient croître considérablement leur dissimilitude avec les employés de bureau. Fort logiquement, avec la catégorie "services", ils enregistrent le plus important rapprochement avec les agriculteurs. Ce dernier rapprochement révèle en partie un encadrement plus important, un équipement, de ces campagnes en mutation.

La dynamique péri-urbaine apparaît donc comme sélective. Elle engage davantage les populations qui disposent du capital social le plus faible. En même temps qu'une substitution urbain-rural, s'opère un tri qui renvoie à une remise en ordre de l'appareil productif, à son développement hors de l'aire bâtie saturée.

Bien entendu, dans ce contexte d'étalement et de reformatage progressif de la ruralité en urbanité, le lotissement des parcelles périphériques et l'équipement des nouveaux logements, comme la constitution d'une trame de services, génèrent leur propre stock de populations ouvrières et commerçantes. Outre les migrations de déconcentration et de décohabitation, la densification et le lotissement périphérique développent des formes d'auto-expansion démographique et économique qui, à leur tour, au-delà d'un certain seuil, produisent de nouveaux débordements, des contagions. Cela pour dire qu'il ne faut pas surestimer le poids des interrelations dans ce processus d'étalement; il faut tenir compte d'une part d'endogène ainsi que du rôle des connexions avec des villes et des villages de province plus éloignés. 
64 un nouveau processus d'intégration urbaine. Il se résume en la possibilité croissante de migrations directes de la province vers les périphéries, alors qu'auparavant, comme le montrent les données migratoires des recensements de 1976 et 1986, le passage par le centre s'imposait ${ }^{38}$. Là encore, l'idée de diffusion du mode de vie urbain mériterait d'être interrogée. Elle prend certainement des chemins détournés, surtout si le centre ne joue plus son rôle de matrice citadine. Cela pourrait nous renvoyer aux analyses de Nabil Bayhum et de Jean-Claude David sur les citadins de seconde zone, en soulignant cependant qu'au Caire, ce serait un processus assez nouveau ${ }^{39}$.

\section{Une urbanité étendue sans transition urbaine}

Entre 1976 et 1986, comme l'indique la régression « taux de variation annuelle de la population totale/taux de variation annuelle de la population active», les actifs ont davantage quitté le centre que ne l'a fait la population dans son ensemble. On assisterait donc à une périphérisation des jeunes actifs ${ }^{40}$. Hypothèse en partie confirmée par l'évolution de l'âge moyen, qui s'organise selon des gradients auréolaires centre-périphérie de plus en plus marqués, s'échelonnant de 38 ans à 18 ans. Pour le moins, l'espace de reproduction de la main-d'oeuvre s'étend. Dans les espaces urbains périphériques, ceux où les agriculteurs comptent pour moins de $20 \%$ et, le plus souvent, pour moins de $10 \%$, la relation entre l'évolution des actifs et l'évolution de la population totale est favorable aux premiers, confirmant bien la logique centrifuge. Mais au-delà, plus loin dans la campagne, la relation s'inverse à nouveau. L'augmentation de la population ne s'accompagne plus d'un mouvement proportionnel des actifs, et cela est plus marqué dans le gouvernorat de Gîza que dans celui de Qaliûbiyya.

Tableau 5: Évolution du poids des six catégories non-agricoles de l'espace rural(plus de $20 \%$ d'agriculteurs) de la région du Caire (\%)

\begin{tabular}{|l|c|c|}
\hline Secteurs professionnels & 1976 & 1986 \\
\hline ingenieurs, techniciens & 5,03 & 7,45 \\
\hline Dirigeants & 3,15 & 3,09 \\
\hline Employes de bureau & 5,38 & 7,63 \\
\hline Gens du commerce & 10,33 & 9,74 \\
\hline Senvices & 10,52 & 13,68 \\
\hline Owriers & 8,99 & 11,34 \\
\hline
\end{tabular}

Même si, globalement, le poids des actifs résidant dans l'espace rural stagne autour de $10 \%$ du total de la région du Caire, leur répartition catégorielle a été modifiée. L'espace agricole péri-mégapolitain compte davantage d'actifs engagés dans le système productif hors agriculture, notamment dans les secteurs industriel et artisanal, de l'ouvrier à l'ingénieur. Là encore, il est difficile de savoir ce qui relève du développement in situ et ce qui engage davantage de flux et d'interdépendances mais, d'une manière ou d'une autre, la circulation et les échanges sont davantage impliqués. Mobilité accrue, multiplication des navettes domicile-travail et localisation plus 
périphérique des nouvelles activités - voire délocalisations en faveur des marges urbaines - sont ici en jeu. Il faudrait en outre développer ce même type de recherche pour l'ensemble du système de villes égyptien afin de distinguer ce qui relève, d'une part, de l'étalement et de la polarisation mégapolitaine et, d'autre part, du processus plus diffus et global d'urbanisation qui concerne la totalité de l'écoumène égyptien (voir tableau 6).

Dans ce champ d'influence irradié par les dynamiques mégapolitaines, un tri social est à l'œuvre, un mouvement structurel qui tient à la résidence de plus en plus périphérique des actifs - de certains actifs, serait-on tenté de penser.S'imposent encore, dans les interstices de cette structuration radio-concentrique, des poches de résistance où la croissance de la population ne s'accompagne pas d'une localisation accrue d'actifs. C'est le cas notamment à Manshiat Nasîr, Mugawarîn ou Dar al-Salâm... Autant de lieux dont l'insertion dans l'espace productif demeure très nettement défaillante. Là se dessinent les contours de la marginalité par rapport à la dynamique structurelle. Cette marginalité n'entre pas dans un schéma classique du type radioconcentrique. Elle relève tout autant des centres, ou de la péri-centralité, que des périphéries. Elle participe d'une complexification et d'une fragmentation de l'espace social.

Tableau 6: Taux annuel de croissance 1976-1986

\begin{tabular}{|l|c|c|}
\hline Secteurs professionnels & "Urbain * & * Rural " \\
\hline Ouviers & 2,67 & 5,37 \\
\hline Ingénieurs, techniciens & 6,25 & 10,78 \\
\hline Employés de bureau & 1,64 & 5,51 \\
\hline Services & $-1,05$ & 1,96 \\
\hline Gens du commerce & 0,58 & $-0,06$ \\
\hline Dirigeants & $-0,16$ & $-0,34$ \\
\hline Total actifs, hors agriculteurs & 2,40 & 4,74 \\
\hline
\end{tabular}

La mégapole, espace de la diversité, de la multiplication des interactions, des effets de proximité comme de la pluralité des activités, reste néanmoins un espace urbain divisé, organisé, voire hiérarchisé. Si la diversité concentrée dans des métriques courtes et des contiguïtés ouvre le champ des possibles, elle multiplie aussi les opportunités de spécialisation. En retour, elle détermine des dissimilitudes plus ou moins volontaires ou contraintes, dépendantes du capital social et culturel des citadins comme de leur date d'insertion, ou encore de leurs relations avec telle ou telle filière économique de production et de distribution.

69 A cette échelle où s'exposent les recompositions socio-spatiales maximums liées à l'étalement, la question du continuum urbain peut être posée. En effet, il s'agit aussi de l'aire des migrations circulaires en relation avec la mégapole. Qr cette dernière est à présent plus étendue que les limites de l'agglomération morphologique indiquant le développement d'un monde entre deux mondes, d'un espace interstitiel où les actifs sont à la fois de la campagne et $\mathrm{d}$ la ville ${ }^{41}$. 


\section{Spécialisations intra-urbaines contre incertitudes périphériques}

70 Ce processus de diffusion de la structure sociale urbaine s'accompagne d'une remise en ordre intra-urbaine, d'ajustements internes. Pour les visualiser, nous prendrons en compte cette conurbation découpée en 333 unités dont la ceinture périphérique regroupe moins de $20 \%$ d'agriculteurs en 1986 . Au total, une aire urbaine de $690 \mathrm{~km}^{2}$ se détache.

71 Cet espace pluriel, non surdéterminé par l'importance des activités agricoles, compte pour un tiers de l'espace total, mais concentre $90 \%$ des actifs hors agriculture. En 1976, cet espace regroupait $89 \%$ des actifs. Existerait-il un effet de captation en partie en contradiction avec l'idée de diffusion du fait urbain? En 1986, à peu de choses près, cet espace correspond à l'agglomération morphologique définie par la continuité du bâti et, à $100 \%$, à celle des années quatre-vingt-dix, plus quelques pôles urbains proches mais non encore conurbés, comme Badrashin (75.500 habitants en 1986) ou Qanâtir (49.400). Il regroupe en revanche relativement moins d'habitants: 80 \%. Il se caractérise par une «sur-concentration »d'actifs qui renvoie à l'accumulation des activités sur un territoire réduit où peuvent aisément se composer des complémentarités et des filières, donc des spécialisations ${ }^{42}$.

\section{Mise à distance versusmixité}

\section{L'émergence des classesmoyennes}

72 De l'évolution de la distribution de la population active dans la mégapole, nous retiendrons surtout le poids croissant des ingénieurs, techniciens, enseignants et professions médicales. Devant les employés de bureau, ils s'imposent dorénavant comme le pivot de cette société mégapolitaine en construction. Ils symbolisent l'émergence possible d'une classe moyenne forgée par l'appareil universitaire à un moment où les autres catégories sont plus stables, voire enregistrent de sensibles reculs. Autrement dit, s'affiche ici l'émergence de la qualité urbaine moderne telle que l'on peut l'observer un peu partout à travers le monde des villes ${ }^{43}$. Une masse incertaine décale les vieilles contradictions urbaines sans toutefois, dans notre cas, que cela se traduise par un fléchissement marqué du poids des ouvriers. Ces derniers sont simplement repoussés plus loin en périphérie (voir graphique 2).

73 Si la libéralisation a autorisé un développement partagé par davantage de familles, c'est autour de cette catégorie symbolisant la classe moyenne qu'il faut le rechercher. En effet, les dirigeants et les commerçants demeurent des groupes fermés assurant à peine leur reproduction d'un recensement à l'autre. Mais entre 1976 et 1986, s'observent surtout des redistributions qui, irrésistiblement, renvoient à l'idée de déclassement social. Les diplômés ne sont pas tous des gagnants de la politique d'ouverture économique: «Oui, de mon temps, c'est à son savoir que se mesurait la valeur d'un homme. Cela suffisait à préserver son existence de la misère et des coups du sort. Du moins en était-il ainsi alors. Mais à présent, avec les mutations qui sont advenues, on assiste à des revers inattendus: la valeur d'un homme s'évalue aujourd'hui à sa fortune, aux biens qu'il a amassés - peu importe comment, du reste. $»^{44}$ 


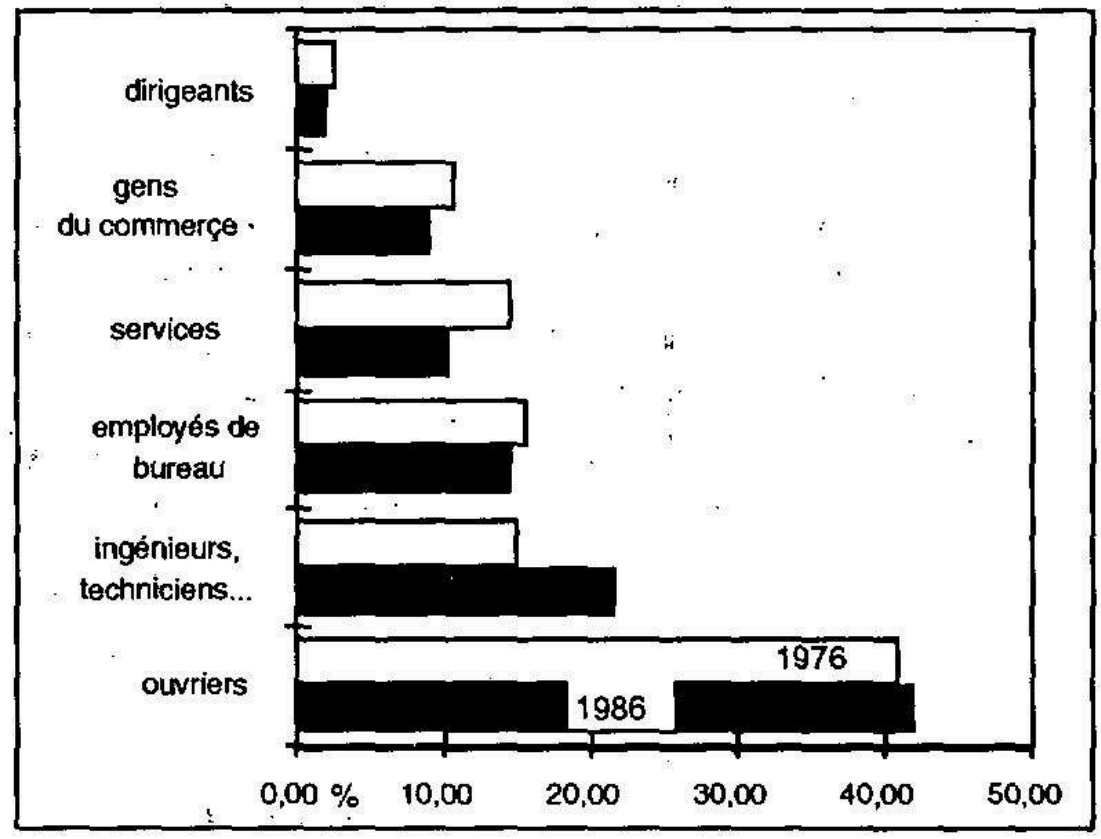

Dans un tel contexte, la catégorie des ingénieurs, techniciens, enseignants et professions médicales s'étire sur l'échelle des positionnements symboliques et géographiques, divisée notamment entre ceux du public et du privé. Se dire ingénieur ne suffit plus pour disposer d'un statut social. Engagé dans le secteur privé, les diplômés du supérieur ont parfois accédé à un standard de vie en accord avec les modes de consommation et d'habitat des classes moyennes supérieures de toutes les grandes métropoles mondiales. Ils ont été en mesure de maintenir leur rang tout en profitant d'un accès accru à l'ensemble des biens et des informations désormais importés ou produits selon des normes transnationales (voitures, équipements ménagers, loisirs, nourriture, réception satellite...). A l'inverse, les ingénieurs, les techniciens et les médecins du secteur public, comme les enseignants, ne peuvent accéder à ce potentiel d'ascension ou d'amélioration du quotidien qu'en s'investissant dans une seconde activité, parfois sans rapport avec leur formation initiale.

Tableau 7 : Indice de concentration intra-agglomération

\begin{tabular}{|l|c|c|}
\hline Secteurs professionnels & 1976 & 1986 \\
\hline ingénieurs, lechniciens & 0,34 & 0,31 \\
\hline Dirigeants & 0,43 & 0,45 \\
\hline Employés de bureau & 0,17 & 0,13 \\
\hline Gens du commerce & 0,15 & 0,14 \\
\hline Services & 0,12 & 0,12 \\
\hline Owriers & 0,27 & 0,29 \\
\hline
\end{tabular}


Il n'en reste pas moins un espace social différencié, où les dirigeants sont encore plus concentrés qu'auparavant, de même que les ouvriers. Le mouvement centrifuge qui affecte ces derniers explique largement leur moindre occupation de l'espace intraurbain. Dans ce contexte, ils ne sont pas en mesure d'investir davantage la ville, voire de s'y maintenir. Ainsi s'établit un processus de distanciation croissante entre les dirigeants, qui consolident leurs positions sans s'étendre, et les ouvriers, dont une partie des localisations traditionnelles les plus centrales sont fragilisées. En revanche, les ingénieurs, techniciens, enseignants et professions médicales sont engagés dans un processus de diffusion qui, en partie, indique le développement à la périphérie d'un vaste espace social mixte. Là se donne à voir le déclassement des ingénieurs, ou l'incapacité d'une partie d'entre eux - les nouveaux diplômés ? - à se glisser dans les quartiers intermédiaires, notamment à Muhandisin ou Madinat Nasr.

\section{Le volontarisme générateur de spécialisation}

76 L'ère nassérienne, en composant au nord et au sud du Caire deux pôles porteurs des espoirs d'une construction nationale populaire reposant sur l'édification d'une " industrie industrialisante ", a consolidé des localisations ouvrières périphériques tant à Hîlwan qu'à Shubra al-Khayma. A Shubra al-Khayma, les ouvriers représentent $56 \%$ des actifs.

L'étalement fonctionnel associé à la massification s'est donc traduit par une spécialisation accrue de l'espace urbain. De même, la vaste cité administrative de Madinat Nasr s'est finalement imposée comme un quartier très spécialisé, puisqu'elle regroupait dès 1986, dans sa partie la plus ancienne, 74 \% d'ingénieurs, techniciens et enseignants, et $46 \%$ de ces derniers dans la partie dont le lotissement n'est toujours pas achevé en 1995. Depuis 1952, les ambitions modernisatrices des présidents Nasser et Sadate ont eu pour effet de distinguer les populations sur lesquelles devaient s'appuyer leur pouvoir et leur projet de société d'abord les ouvriers, ensuite les classes moyennes. Mais ces actions publiques auront surtout contribué à produire des espaces spécialisés susceptibles de contrarier les vertus urbaines associées à la mixité. Les villes nouvelles, malgré les intentions affichées, s'orientent vers la même logique de spécialisation, se faisant l'écho de conceptions très fonctionnalistes. La croissance démographique s'est accompagnée d'un saut qualitatif : il n'y a plus un profil social du Caire plus ou moins centré et associé à des micro-spécialisations par rue, mais des profils périphériques très marqués.

78 Ce mélange inégal peut se cartographier globalement sur la base d'un coefficient de spécialisation qui varie entre 1 et $0^{45} .1$ indique une spécialisation totale dans une catégorie et 0 une mixité proportionnelle à la distribution moyenne des 7 catégories socio-professionnelles. Ainsi s'expose de l'urbain ordonné et ses limites marquées par le maintien d'une spécialisation centrée sur le travail des champs (voir carte 6 et tableau 8 pages suivantes). 


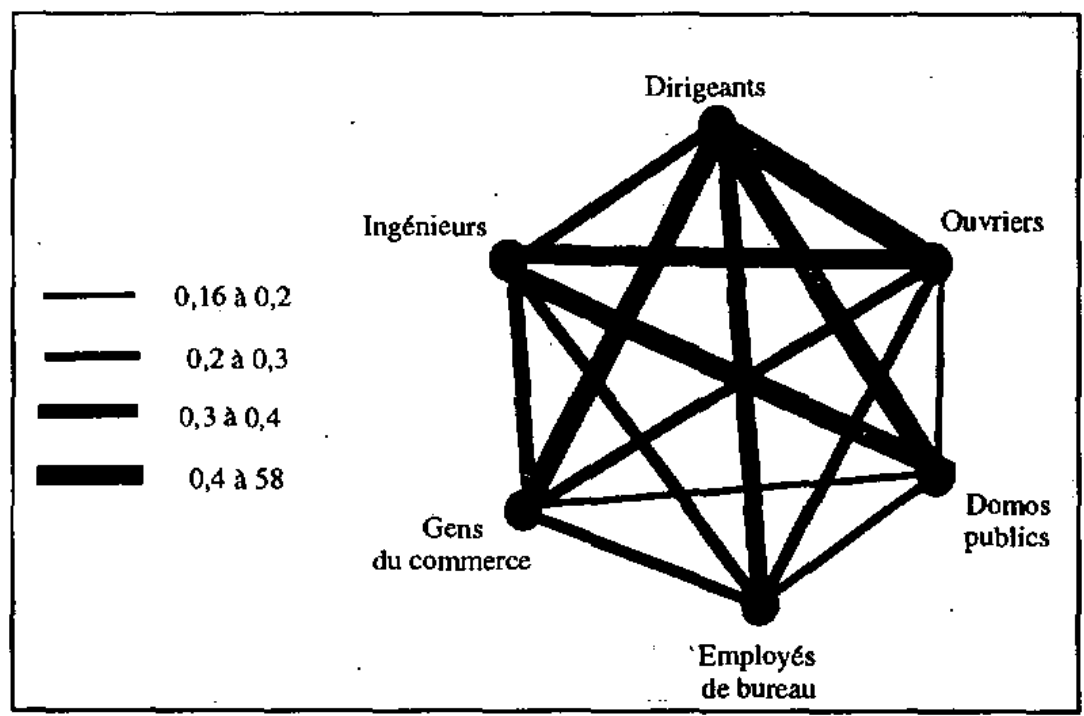

\section{Les faubourgs et la rupture des classes moyennes}

La croissance importante du nombre des ingénieurs, techniciens et enseignants s'est donc en partie traduite par une diffusion périphérique vers les nouveaux quartiers construits à l'est sur le désert (voir carte 7). A l'opposé, avec les ouvriers, ils ont aussi gagné des quartiers d'habitat sous-standard à la légalité incertaine : les faubourgs. La, ils sont simplement en mesure de maintenir une relative distinction à l'échelle de la rue ou de l'immeuble, dans un moment où les images se sont brouillées entre ceux qui ont pu suffisamment capitaliser, notamment par une migration dans le Golfe - ouvriers ou ingénieurs - et les autres (en 1994, selon la Capmas, 2 millions d'Égyptiens travaillaient à l'étranger). Bien entendu, d'aucunsse sont maintenus, voire affirmés, en compagnie ou à proximité des dirigeants, à Muhandisîn plus qu'à Zamalek. 
Carte $n^{\circ} 6$ : Le décentrement des diplômés entre 1976 et 1986

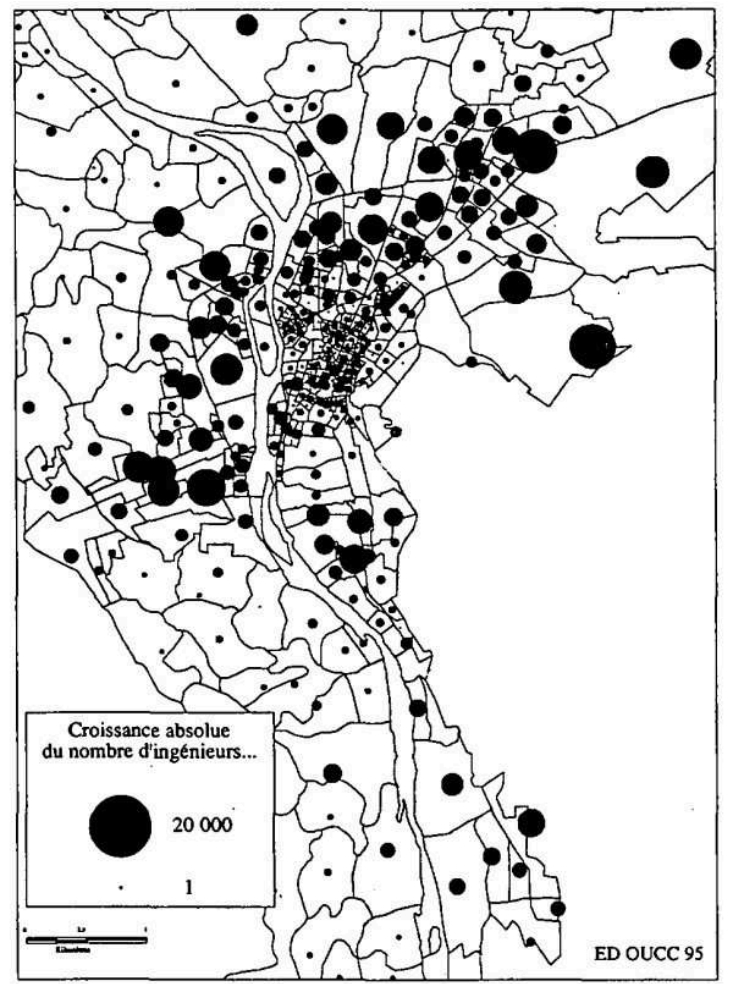

Tableau 8 : Matrice de la dissimilitude socio-professionnelle infra-agglomération

\begin{tabular}{|l|l|l|l|l|l|l|}
\hline & & & & & 1976 & \\
\hline $\begin{array}{l}\text { Secteurs } \\
\text { professionnels }\end{array}$ & $\begin{array}{l}\text { Ingénieurs } \\
\text { techniciens }\end{array}$ & Dirigeants & $\begin{array}{l}\text { Employés de } \\
\text { bureau }\end{array}$ & $\begin{array}{l}\text { Gens du } \\
\text { commerce }\end{array}$ & Services & Ouvriers \\
\hline $\begin{array}{l}\text { Ingénieurs, } \\
\text { techniciens }\end{array}$ & - & 0.17 & 0,22 & 0,37 & 0,32 & 0,45 \\
\hline Dirigeants & 0,23 & - & 0,37 & 0,48 & 0,44 & 0,56 \\
\hline $\begin{array}{l}\text { Employés de } \\
\text { bureau }\end{array}$ & 0,22 & 0,42 & - & 0,23 & 0,19 & 0,27 \\
\hline $\begin{array}{l}\text { Gens du } \\
\text { commerce }\end{array}$ & 0,30 & 0,47 & 0,17 & - & 0.19 & 0,20 \\
\hline Services & 0,28 & 0,47 & 0,18 & 0,16 & - & 0,19 \\
\hline Ouvriers & 0,40 & 0,58 & 0,24 & 0,20 & 0,17 & - \\
\hline & 1986 & & & & \\
\hline
\end{tabular}


Carte $n^{\circ} 7$ : Les spécialisations socio-professionnelles en 1986

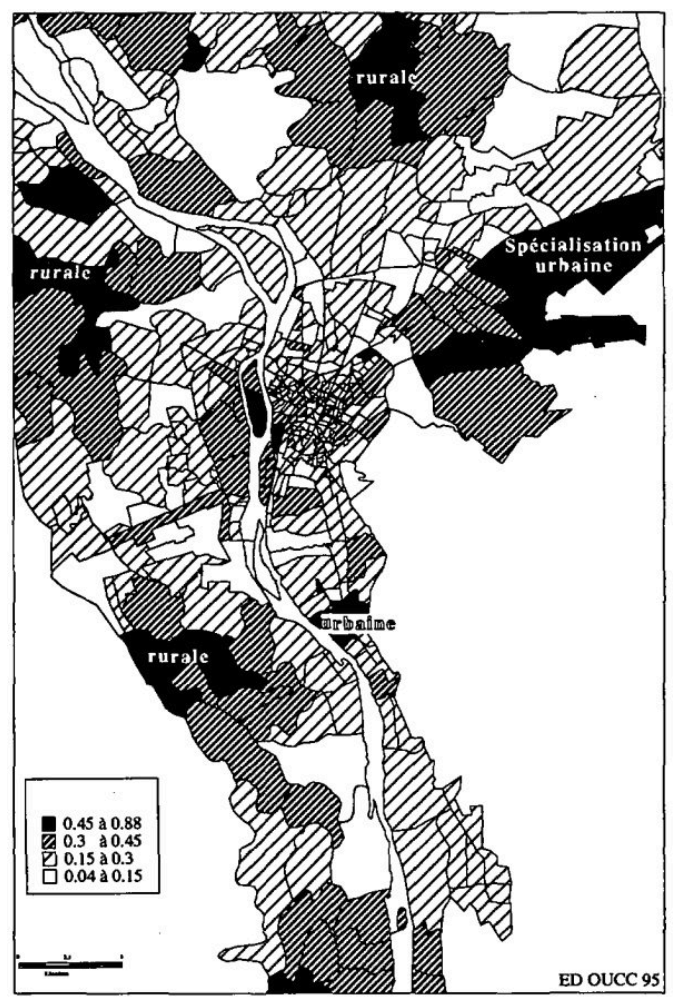

80 Cet espace de mixité croissante résulte donc de la fragilisation de ce que l'on serait tenté d'appeler la classe moyenne, et au débordement du modèle central vers les faubourgs non planifiés. Il est associé à l'étalement, mais à un étalement minimum compte tenu de la résistance des fortes densités. Dans ces quartiers qui regroupent la moitié des habitants du Caire, se compose encore une urbanité centrée, mixte, c'est-àdire de la diversité dans des métriques de proximité. L'insertion de cette diversité ou son maintien marginal dans la qualité de sous-standard extra-légal est l'enjeu du moment. La peur de cette mixité interprétée comme du désordre a conduit jusqu'à présent à son contournement et à la production d'espaces fonctionnels qui ne sont pas en mesure d'assumer la totalité de l'extension du fait urbain. Ces unités spécialisées facilitent surtout le contrôle social, le tri et l'encadrement, de même que les performances du système de production. Du fait de leur mixité sociale et de la coexistence entre les ateliers, les commerces et le logement, ils répondent parfaitement à la définition de faubourg ${ }^{46}$. Le faubourg, c'est le lieu où des populations diverses qui n'ont pas accès au bourg, au centre de la ville, composent des quartiers populaires, souvent en construisant leur propre logement ou en se regroupant par rues.

81 Toutefois, le potentiel d'urbanité de cette mixité relative est a priori contrarié dans la mesure où elle ne représente pas, comme l'insertion dans les quartiers populaires centraux et péri-centraux, une aspiration mais, au moins en partie, une relégation. Ces quartiers ne disposent pas, ou pas encore, du prestige social qu'ont su conserver des quartiers centraux en partie dégradés. Ils sont davantage désignés comme des lieux étrangers à la matrice urbaine. Néanmoins, avec l'essor d'un nouveau quartier, des individus ont pu affirmer leur position sociale et s'imposer comme notables locaux. Leur avenir est désormais associé à celui de leur quartier, car leur prestige n'est pas aisément transférable ailleurs, ils constituent de fait une population qui vit plutôt bien 
l'essor des faubourgs du Caire. Un certain nombre de verrous physiques, l'absence d'une voirie clairement hiérarchisée ou de transports publics, matérialisent la relégation. Toutefois, les services compétents ont entrepris des travaux de percement et de franchissement qui laissent à penser quecela pourrait changer et se hiérarchiser. Le long de ces avenues désormais reconnues, des immeubles rappelant Muhandisîn se dressent déjà et les spéculations vont bon train. A l'inverse, répondant à des directives de mise en ordre, la fermeture par la police (hiver 1994-95) d'un certain nombre de marchés à Bûlâq alDakrûr (Abu Qatâda) et Imbâba (Sûq aI-Gum'a et Sûq al-Gamal) contribue à réduire l'intégration de ces quartiers et à détruire des centralités plus ou moins spontanées et héritières des noyaux villageois. L'avenir de cette diversité périphérique est pour le moins incertain.

82 Ce possible rapprochement entre le centre et les quartiers spontanés renvoie encore à ce que Philippe Panerai et Galila el Kadi ${ }^{47}$ ont pu relever comme analogies morphologiques. Ces formes urbaines sont toutes deux cadencées par des métriques pédestres, c'est-à-dire par des dimensions qui, quelles que soient les distances sociales internes, imposent de multiples situations d'interactions à travers des ruelles, des impasses et des cours. Ces conditions de relative mixité impliquent, presque autant dans les nouveaux faubourgs que dans la vieille ville, une insuffisance d'infrastructures publiques. S'y retrouveraient encore des similitudes dans la manière de se structurer " en grappes ", de s'insérer dans des réseaux largement fondés sur la famille tels que les a mis en évidence Diane Singerman pour la vieille ville du Caire ${ }^{48}$. Le lien entre ces deux tissus morphologiques et sociaux tient aussi à l'enrichissement des réseaux familiaux entre les enfants installés dans les nouveaux faubourgs et les parents résidant dans les vieux quartiers populaires. Cette diversité pourrait renvoyer à ce que Jacques Berque désignait comme «l'humanité citadine qui jadis formait un tout» ou, si l'on osait le saut historique, à l'absence de cloisonnement social relevé par Sylvie Denoix pour Fustât-Misr aux Xllle-XIVe siècles ${ }^{49}$.

Ailleurs, hors du centre et des nouveaux faubourgs, la spécialisation l'emporte selon trois polarités : ouvriers ; puis ingénieurs, techniciens et enseignants ; enfin, dirigeants. Malgré la montée en puissance des classes moyennes, des cols blancs qualifiés, Le Caire contemporain apparaît moins centré sur une mixité qu'éclaté entre des espaces fonctionnels et une mixité périphérique aux contours flous. On peut se demander si cette dernière représente ou représentera l'avenir de l'urbanité, c'est-à-dire l'extension périphérique du noyau central, ou si elle marque une rupture avec l'idée de ville. N'estce pas davantage une relégation ou un déclassement et, par là même, une remise en cause du modèle urbain intégrateur ? Là comme ailleurs, tous les diplômés ne trouvent pas à s'insérer de façon avantageuse dans un univers urbain où toutes les aspirations paraissent pourtant si proches. Exclusion peut-être ? L'avenir de ces quartiers dépend en partie du regard porté sur eux, de la manière de les désigner et d'y intervenir. La soumission formelle de ces quartiers en termes d'intégration économique aurait pour effet de fragiliser le tissu social et de remettre en cause la mixité. Une stricte rationalité économique évincerait les plus démuniset introduirait de nouveaux blocages, elle déstabiliserait la dynamique et introduirait des charges nouvelles. En ce sens, la notion de faubourg n'est pas à interpréter selon une logique évolutionniste, comme un moment de l'histoire de la ville condamné à disparaître au profit d'autres formes urbaines, par exemple des banlieues ordonnées et spécialisées. 


\section{Des axes structurants : densité, commerce et pouvoir}

Notre objet n'est pas d'arriver à une synthèse en quelques types bien assurés et aux contours précis, mais simplement de qualifier Le Caire à travers la mosaïque des quartiers qui le composent. Le flou subsistera. La complexité mégapolitaine ne pourrait se satisfaire de l'assurance d'une délimitation en quelques unités homogènes. En outre, nos matériaux sont insuffisants pour statuer ainsi sur l'hétérogénéité de l'espace intraurbain.

Un zonage assuré pourrait aussi faire oublier toute la richesse des interrelations. La résidence n'enferme pas les citadins, car la mégapole est aussi un espace où se concentrent des potentiels toujours croissants de mobilités. Au fond, en dessinant les contours de la diversité urbaine à travers ces catégories socio-professionnelles enregistrées au lieu de résidence, nous ne donnons à voir que « la ville où l'on dort " ${ }^{50} . \mathrm{Il}$ n'en reste pas moins vrai que la résidence demeure le lieu de l'appartenance et de la distinction sociale : nous nous déplaçons avec cette identité.

Sur la base d'une cartographie des quotients de localisation, nous pouvons néanmoins mettre en évidence quelques axes structurants. Ce rapport entre la distribution d'une catégorie dans une unité et sa distribution moyenne dans la totalité prise en compte met en valeur sa concentration ou sa dispersion ${ }^{51}$.

\section{Carte $n^{\circ} 8$ : Les ouvriers en 1986}

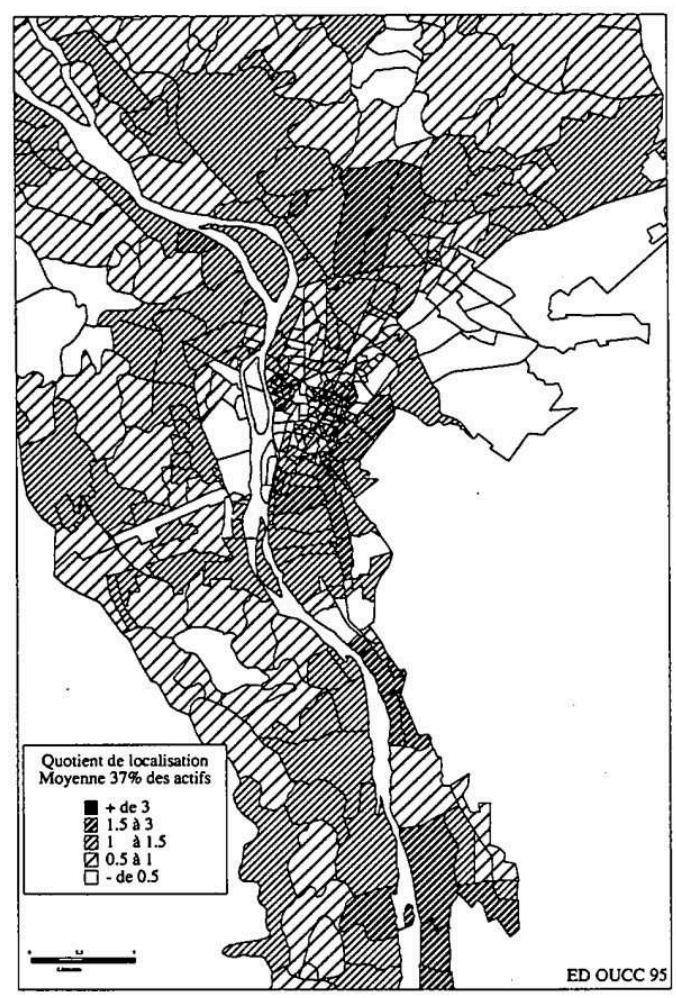


Carte $\mathrm{n}^{\circ} 9$ : L'axe des dirigeants en 1986

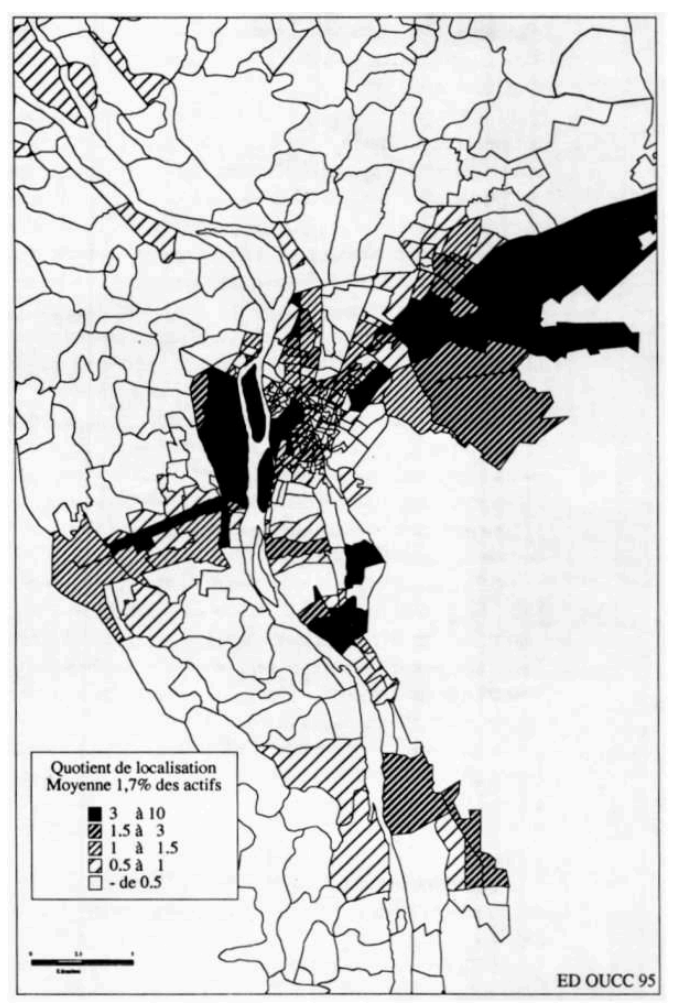

Carte $n^{\circ} 10$ : Les commerçants en 1986

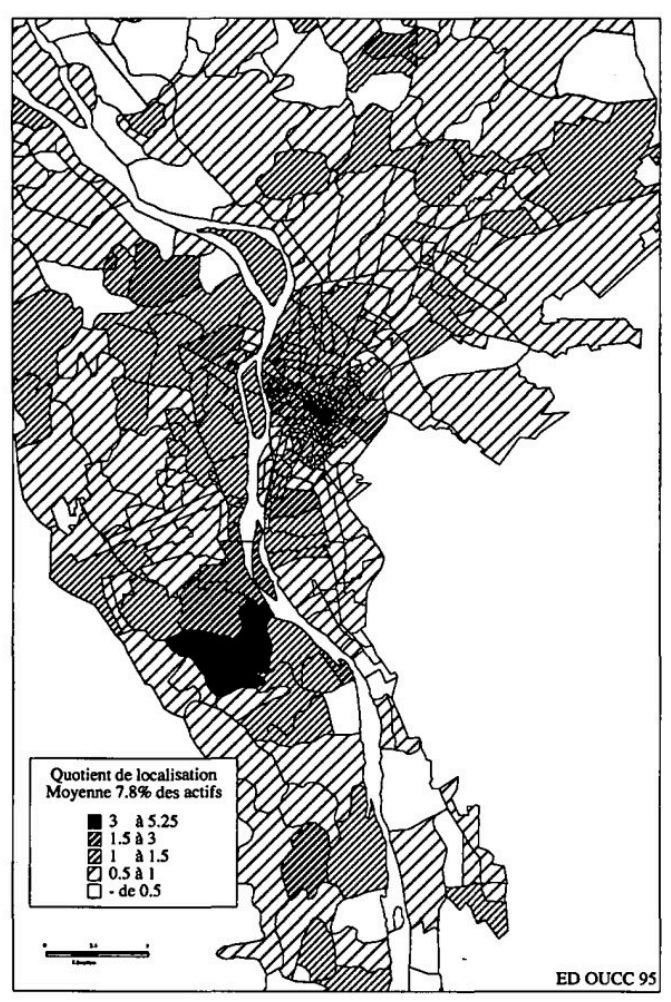

L'examen de la carte 9 concernant les catégories des dirigeants permet de mieux comprendre cette mise en valeur de la distribution. La valeur 1 correspond à la 
distribution moyenne dans la région du Caire de la catégorie cartographiée, 0,5 à la moitié de la moyenne, et 2 à deux fois plus que la moyenne. Plus la part d'une population est faible, plus son potentiel de concentration est élevé. Cette qualité théorique est mise en pratiquepar les dirigeants, population la moins nombreuse : 1,7\% des actifs. Ils s'imposent en effet comme les plus regroupés (quotient de localisation maximum : 10,1), soit $17 \%$ de la population active à Qasr al-Nîl. Cela en fait aussi la plus absente dans la majorité des unités.

Inversement les ouvriers, compte tenu de leur poids (37\% des actifs) occupent forcément davantage d'espace (carte 8). Toutefois, ils sont largement absents des unités où se concentrent les dirigeants - y comptant pour moins de $15 \%$ des actifs - alors qu'ils occupent à plus de 50 \% les zones industrielles de Hîlwan, Tûra au sud et Shubrâ al-Khayma au nord. Basatîn, Dâr al-Salâm ou Khâlifa ont beaucoup plus à voir avec les activités manufacturières centrales et péri-centrales, plutôt artisanales ou protoindustrielles. Dans les zones d'urbanisation dite spontanée comme Bûlâq al-Dakrûr ou Imbâba, le poids des ouvriers n'est pas supérieur à la moyenne. Ces espaces conservent donc bien une certaine mixité. De plus, aucune autre catégorie n'y est surreprésentée.

Ces distributions, en décomposant la cartographie de la spécialisation de l'espace, donnent à voir deux axes structurants, l'un dessiné par les dirigeants et l'autre par les commerçants. Les dirigeants se distribuent en effet de part et d'autre d'un axe fonctionnel essentiel qui permet l'interconnexion est-ouest, de l'aéroport au plateau des Pyramides en passant par l'avenue du même nom et l'artère en partie suspendue du Six-Octobre (carte 9). Les immeubles de standing des avenues al-Ahrâm ou al-Faysal à Giza s'inscrivent dans une relative continuité avec ceux de Zamalek ou d'Heliopolis. Au total, ils dessinent comme un barrage sur le Nil, donc en travers de l'orientation traditionnelle, Il pourrait être interprété comme l'inscription matérielle du contrôle sur le cours des choses. Sur cet axe, l'essentiel est capté, drainé, échangé, attribué et capitalisé. D'un strict point de vue fonctionnel, il permet l'interconnexion entre les centres névralgiques, le pays et les moyens de communications internationaux. Compte tenu de la centralisation administrative et économique, cette classe dirigeante représente plus qu'une élite régionale: elle s'impose comme le pouvoir en Égypte. En outre, si l'agglomération du Caire, en 1986, regroupait à peine $20 \%$ de la population urbaine de l'Égypte, elle concentrait près de $50 \%$ de ses dirigeants.

A présent, cette disposition réticulaire du pouvoir tend à se prolonger vers les villes nouvelles où se concrétisent de luxueux programmes de villas. La référence à une centralité aujourd'hui obsolète, avec le programme " New Garden City » de la ville du Six-Octobre, symbolise très bien ce processus de décentrement et de remise en cause de l'immédiateté matérielle au profit de la mobilité et de la communication. C'est aussi un indicateur de mutation urbaine par rapport aux référents classiques qui veulent que dans la métropole du Tiers-monde, l'élite, si elle n'occupe pas exactement le centre, reste à proximité, notamment pour bénéficier dans les meilleures conditions des équipements élémentaires (eau, électricité..). Bien entendu, ces développements ne remettent pas en cause le statut des berges du Nil, tout à fait favorableà l'ostentation. Mais la saturation s'étendant, une localisation plus périphérique devient attrayante. De plus, l'extension des réseaux sur terrain vierge devient plus performante que la modernisation de la desserte de quartiers péri-centraux de plus en plus saturés. Cette extension réticulaire est encore à mettre en correspondance avec un changement de métrique qui recompose les distances et les formes selon les capacités du véhicule 
personnel, désormais plus accessible, et en fonction de la diffusion des moyens modernes de télécommunication.

91 En revanche, la centralité demeure toujours très marquée en ce qui concerne la résidence des commerçants et du commerce lui-même, du fait de la persistance d'une proximité entre la résidence et l'activité (carte 10). Cette centralité commerciale n'est plus celle de la vieille ville. Elle n'accompagne plus l'axe historique des fortes densités qui, longtemps, a organisé l'activité marchande. Elle forme au contraire un axe perpendiculaire, intégrateur, associant les sites anciens du commerce spécialisé, les sûq, et la ville planifiée qui s'étend vers les berges du Nil : se retrouvent encore des pôles périphériques, des marchés de gros agricoles en bordure des zones qui résistent le mieux à l'urbanisation, notamment à Abû Numrûs au sud de Giza.

\section{Fractionnement versus mobilité}

92 En étalement, l'agglomération mégapolitaine s'altère tout autant qu'elle urbanise son environnement. Dans un contexte de croissance démographique encore fort, un décalage relatif de la distribution socio-professionnelle, des filtres et des aspirations engage des redistributions tant en son cœur que vers des aires spécialisées et des faubourgs incertains, Il existe donc bien une structure et des contradictions spatiales qui n'autorisent pas à parler d'homogénéisation. Aucune réduction des distances spatiales entre les catégories socioprofessionnelles les plus éloignées n'est observée. Simplement, dans un contexte de fragilisation généralisée, une certaine mixité péricentrale s'est développée, mais elle reflète peut-être davantage une relégation que l'essor d'une citadinité élargie. Du point de vue strictement social, l'affirmation des classes moyennes éduquées sur la scène urbaine constitue un fait majeur qui s'est notamment traduit par le brouillage de l'opposition spatiale ouvriers-dirigeants, même si cette dernière n'a fait que s'accentuer. Elles ont introduit de l'incertitude, même si l'essentiel se tient etsi l'ordre social semble satisfait.

93 Au total, la sectorisation se cristallise, elle théâtralise les rapports sociaux et amplifie les logiques de distinction. La mixité est remise en cause et la centralité n'apparaît pas clairement. L'idée de ville est donc fragilisée ou, pour le moins, se décale, autrement dit elle se réinvente. En partie dégradée et saturée, la centralité encombre. Elle n'est plus une nécessité fonctionnelle dans un contexte ou la ville centrée ne polarise plus les activités de production porteuses, voire même la totalité des fonctions de commandement. Dans le même temps, une logique de filières et de mise en réseaux interconnecte des espaces spécialisés périphériques pilotés par un ensemble de flux tant immatériels que matériels. La ville fuit ses polarités les plus ancrées mais aussi les plus lourdes. Ainsi, les zones industrielles des villes nouvelles autour du Caire concentrent $20 \%$ des exportations de biens manufacturés de toute l'Égypte. Si les axes et les nouvelles artères recomposent la mégapole sur la base d'un accrochage aux pôles performants implantés sur les plateaux désertiques, son insertion au système-monde constitue un méta-réseau structurant. C'est à travers cette interconnexion du local au global que Le Caire peut être qualifié de ville-monde. Mais cette dynamique ne résout pas les déchirements internes, voire les amplifie avec le rôle croissant d'une circulation économique extravertie qui s'appuie davantage sur des complémentarités exogènes que sur des ressources locales. A la ville compacte se substitue de l'urbain réticulé et décentré inscrit dans des logiques de filière. 

faubourgs para-légaux, une résistance du compact et de la mixité dans des métriques de proximité continue de se développer, en contradiction, sans doute, avec la ramification, l'expansion réticulaire et la spécialisation selon des métriques réglées par les déplacements automobiles. Dans ce contexte, la mixité des faubourgs et les scories d'une diversité centrée peuvent en partie être vues comme l'expression matérielle de la résistance du territoire contre l'espace flux. Nous pouvons nous risquer à voir dans ces espaces péricentraux denses, mixtes, des terrains favorables à l'affirmation identitaire, s'opposant à la société ségrégative de l'espace en réseau. Cette opposition, certes des plus réductrices, peut néanmoins être gardée à l'esprit dans la mesure où la construction sociale de ces faubourgs et la production matérielle qui en fait partie autorisent une appropriation plus profonde que les quartiers planifiés et plus spécialisés. Il convient aussi de retenir que Le Caire demeure l'une des villes les plus denses du monde et que son étalement reste très faible, incapable de satisfaire pleinement les échanges.

Mais encore une fois, ce travail sur les localisations ne dit rien de la circulation, des échanges et de la mobilité. Autrement dit, il ne permet pas de dire que tel ou tel processus ou telle construction identitaire vient d'ici ou de là, car les interrelations génèrent tout autant des dynamiques que les lieux. De plus, ce contexte d'étalement, ou plus exactement, d'étirement qui caractérise Le Caire engage un accroissement des mobilités. Si la ville peut être définie par le couple densité plus mixité, la mégapole se caractérise par l'intensité de la circulation. Cette accélération des mouvements circulatoires est consécutive de son décentrement et de sa fragmentation. La mégapole n'est plus tout à fait une ville, en ce sens qu'elle n'est plus un terrain où les rapports sociaux se cristallisent autour d'un point zéro qu'il faut contrôler, ou des efforts pour se maintenir à proximité ou se rapprocher du centre. La lutte contre la distance s'efface en partie au profit de la maîtrise des flux ou, simplement, de l'intégration à cette société en réseaux.

\section{ANNEXES}

\section{« occupations » ou catégories socio-professionnelles}

\section{Groupe 1 - Ingénieurs, techniciens et enseignants}

Scientifiques, ingénieurs, techniciens chimistes et physiciens

Architectes, urbanistes, ingénieurs et techniciens électroniciens, mécaniciens, métallurgistes, chimistes....

Pilotes d'avion, ingénieurs, techniciens aéronautiques, officiers, ingénieurs et techniciens marine marchande 
Ingénieurs et techniciens biologistes, zoologistes et agronomes

Médecins, dentistes, vétérinaires, pharmaciens et assistants

Statisticiens, mathématiciens, économistes, comptables

Juges et avocats

Enseignants du primaire, du préparatoire (collège), du secondaire et du supérieur

Ministres du culte

Journalistes, critiques et écrivains

Artistes, sportifs

Bibliothécaires, sociologues et anthropologues.

\section{Groupe 2 - Dirigeants}

Membres des services gouvernementaux (Haute administration publique), gouverneurs...

Directeurs généraux (public ou privé), directeurs d'entreprise sauf exploitation agricole

\section{Groupe 3 - Employés de bureau}

Chef de bureau, secrétaires et autres

Personnels des services gouvernementaux

Dactylographes, informaticiens, documentalistes, archivistes

Chefs de gare, de bureau de poste et postiers, téléphonistes...

Chauffeurs relatifs aux activités de bureau

\section{Groupe 4 - Gens du commerce}

Travailleurs, dirigeants grossistes ou détaillants et employés salariés ou non

Dirigeants d'un établissement commercial, commerçants, acheteurs, techniciens de vente, représentants de commerce

Commerce des assurances

Vendeurs, vendeurs de rue, vendeurs de journaux...

\section{Groupe 5 -Travailleurs des services}

Propriétaires et dirigeants d'une société de services et employés

Services domestiques, domesticité, cuisiniers

Services de nettoyage

Laverie, coiffeurs

Services de protection civile, pompiers, policiers, gardiens 
Guides

\section{Groupe 6 - Agriculteurs et pêcheurs}

Propriétaires et travailleurs

\section{Groupe $7(8,9)$ - Ouvriers de l'industrie, du transport et du bâtiment}

Contremaîtres, ouvriers salariés et journaliers

Mineurs, métallurgistes, chimistes... (tous secteurs)

Tanneurs, tailleurs (textiles), dockers

Bâtiments : peintres, maçons...

Chauffeurs routiers, de trains etde charrettes

Source : Capmas, Caractéristiques de la population, recensement de 1986, $2^{\mathrm{e}}$ vol., réf. $873 / 91 /$ cac.

\section{NOTES}

1. Kadaré I., Chronique de la ville de pierre, Hachette Littérature, Paris, 1973.

2. Ben JellounT., « Amère fiction du Caire », Le Monde, 03/12/93.

3. Haeringer $\mathrm{Ph}$., « La mégapolisation du monde, du concept de ville à la réalité des mégapoles », Géographie et culture, 1993, p. 3-14.

4. Raymond A., Le Caire, Fayard, Paris, 1993 (428 p.), p. 369.

5. El Kadi G., «La division sociale de l'espace du Caire : ségrégation et contradictions », MagbrebMachrek $\mathrm{n}^{\circ} 110,4^{\mathrm{e}}$ trimestre 1985, p. 35-55.

6. Ilbert R., "Implosion », Le Caire, Autrement hors série $n^{\circ} 12$, 1985, p. 150-157. Cette idée d'implosion avait déjà retenu l'attention d'Yves Lacoste dès 1983 (Éditorial, "L'implosion urbaine ", Hérodote $\mathrm{n}^{0} 31$, p. 3-8).

7. Volait M., «Composition de la forme urbaine du Caire », Peuples Méditerranéens n0 41-42, 1988, p. 105-118.

8. Cf. notamment IAURIF-GOPP, Implementation of the Homogeneous Sector Concept, 4 vol., Le Caire, 1988.

9. Lereshe J. P., Joye D., « Métropolisation de l'urbain au politique », Espaces Temps, Les Cahiers n 51-52, 1993, p. 6-17.

10. Mela A., «Sociologie du territoire : alternative au post-modemisme », Flux $\mathrm{n}^{\circ} 8$, avril-juin 1992, p. 5-15.

11. Nwokolo B., The Application of Western Urban Theories in Middle Eastern Cities: A Case Study of Cairo, PhD, Boston Univeresity, 1978, 200 p. (Si l'auteur, tout au long de son travail, pose le postulat de la particularité des villes arabes, notamment la prédominance des relations de face à face, il ne réalise pas, en fin de compte, ce qu'il avait projeté de faire, c'est-à-dire tester les modèles énoncés par l'école de Chicago. S'en tenant à son idée d'un «islam facteur d'homogénéité », il semble demeurer dans une ville hors du temps et "à la stratification traditionnellement basée sur l'ethnicité et l'appartenance religieuse ».)

12. Diachronique, cette étude suppose l'utilisation d'unités géographiques dont la délimitation demeure constante durant la période prise en compte. Aussi, là où, entre 1976 et 1986, il y a eu subdivision d'une unité en deux ou déplacement des limites d'une unité ou de plusieurs unités, 
les données ont été agrégées dans les limites de 1976 (cela suppose l'utilisation du maillage le plus fin, c'est-à-dire de l'échelle des qura et shyakha, ou village et quartier). Notre fonds est donc celui du recensement de 1976, avec parfois des objets qui regroupent deux unités de 1976 quand la limite d'une d'entre elles a bougé.

13. Dewey R., "The Rural-Urban Continuum: Real but Relatively Unimportant ", American Journal of Sociology, LXV1,1960, p. 60-67. Les notions de mixitéet d'hétérogénéité sont toujours centrales, notamment chez Jacques LÉVY dans ses travaux sur le thème « Ville-Europe »: "Si nous définissons aujourd'hui la ville (mais on pourrait dire cela avec d'autres mots) comme une situation spatiale caractérisée par la concentration d'une société en un lieu $(d=0)$ en sorte d'y maximiser la densité et la diversité des interactions sociales", la ville est en ce sens une des options mobilisées par les sociétés pour lutter contre la distance. Voir «Urbanité : à inventer. Villes : à décrire ", Les Annales de la recherche urbaine, 64, 1994, p. 11-16.

14. Les CSP anglo-saxonnes n'ont qu'un rapport lointain avec les CSP françaises, dont la qualité et la complexité ont peu d'équivalent de par le monde (cf. Desrosières A, Thévenot L, Les catégories socio-professionnelles, coll. Repères $n^{\circ}$ 62, La Découverte, 1992, 125 p.). A l'échelle plus agrégée des qism et des markaz, des tableaux croisés permettent de composer des catégories se rapprochant davantage de nos CSP, mais ce travail n'est pas exposé ici ; nous avons privilégié la mise en évidence de l'agencement géographique de la diversité sociale, les nuances spatiales, à l'échelle la plus fine des shyakha et des qura.

15. La définition de la huitième catégorie regroupant les activités non définies ayant fluctué entre 1976 et 1986, sans pour autant affecter les 7 autres classes, cette catégorie n'a pas été prise en compte ; en particulier, les actifs étrangers y ont été rajoutés alors qu'ils étaient comptabilisés dans les inactifs en 1976, de même que les jeunes n'ayant jamais exercé d'emploi.

16. Waterbury J., The Egypt of Nasser and Sadat, Princeton University Press, 1983, 475 p.

17. Park R. E., «La ville. Propositions de recherche sur le comportement humain en milieu urbain ", The City, Burgess, Park, MacKenzie, 1925 (traduit dans Grafmeyer Y., Joseph I., L'École de Chicago, Aubier, 1990, p. 83-130).

18. Abu Lughod J., Ezz al-Din A., Cairo Fact Book, Cairo Social Research Center, American University of Cairo, 1963. Janet Abu Lughod a construit son œuvre avec le souci permanent de discuter les méthodes el les concepts alors - et toujours - en débat dans les sciences sociales. Elle s'est notamment appuyée sur les riches pages léguées par ce qui a été désigné comme l'École de Chicago et sur les outils mobilisés dans les années cinquante-soixante pour discuter les hypothèses des pionniers, c'est-à-dire les méthodes de l'écologie urbaine (Abu Lughod J., "Testing the Theory of Social Area Analysis: the Ecology of Cairo, Egypt ", American Sociological Review, vol. 34, 1969. p. 198-212). Ces travaux avaient pour ambition de relever et d'expliquer les phénomènes sociaux qui accompagnaient le passage de la population urbaine nord-américaine de 300.000 personnes en 1800 à quelque 40 millions en 1910. Cependant l'analyse écologique est parfois un outil contestable qui a mené à maintes conclusions abusives (" ecological fallacies »), sur la base de relations qui, a priori, n'étaient que des co-occurences spatiales. Ses travaux s'inscrivaient dans un programme du département de géographie de l'université de Chicago qui visait à tester théories et méthodes dans des villes non occidentales ("systematic cross-cultural analysis »\}. Outre Le Caire, l'approche la plus aboutie fut conduite par Brian Berry à Calcutta (Berry B, Ress P., " The Factorial Ecology of Calcutta ", American Journal of Sociology n ${ }^{0} 74,1969, \mathrm{p}$. 445-91 ; Berry B., Kasarda J., Contempory Urban Ecology, New York, Macmillan, 1977, p. 156). Il faut aussi retenir comme référence égyptienne le $\mathrm{PhD}$ de 'Abd al-Latif 'Abd aI-Hamîd sur Alexandrie, soutenu en 1970 à l'Ohio State University (The Ecological and Social Structure of Alexandria, an Examination of Urban Subarea Data1947and 1960) ; voir également 'Abd al-Latif A. H., «Factor Structure and Change Analysis of Alexandria 1947 and $1960 »$. Kent P. Swirian éd.. Comparative Urban Structure Studies in the Ecology of Cities.Ohio University Press, 1974, p. 338-49. Plus 
récemment, Ameur W., Écologie d'une ville arabe: dynamique des structures internes du Caire (1947-1986) (en arabe), doctorat, Université du Caire, 1990,2 vol.

19. Clerget M., Étude de géographie sociale et d'histoire économique, Imprimerie E. R. Schindler, Le Caire, 1934, 2 vol. (cet ouvrage contient un important travail de cartographie de la distribution sociale des populations à partir des données du recensement de 1927).

20. Wirth L, « Urbanism as a way of life », American Journal of Sociology, XLIV, 1938, p. 1-24 (traduit dans Grafmeyer Y, Joseph I., op. cit., p. 255-281).

21. Abu Lughod J.., « Migrant Adjustment to City Life: the Egyptian Case », The American Journal of Sociology, XLVII, 1961, p. 22-32 ; voir aussi : Petersen K. K., «Villagers in Cairo: Hypotheses vs. Data», The American Journal of Sociology, $\mathrm{n}^{\circ} 77-3,1971$, p. 56-73. La ruralisation semble ne plus être qu'une inertie médiatique (par exemple, «la ville nouvelle d'Héliopolis (...) est, elle aussi, surpeuplée par les émigrants ruraux » (Kepel G., «Le Caire », GéoMagazine, octobre 1994, n 188). En revanche, à présent, la recherche sur Le Caire s'énonce davantage dans des termes comparables à ceux qui suivent ; «II y a des quartiers dans lesquels se mélangent ruralité et culture ancienne du Caire pour créer, socialement et physiquement, de nouvelles formes urbaines. » (Tekçe B., Oldham L., Shorter F., A Place to Live: Famines and Child Health in a Cairo Neightborhood, American University in Cairo, 1994, 201 p.) ; ou encore ; "Tout éloigne les conditions de production de ces quartiers d'un modèle traditionnel ou rural. Or, l'échelle de ces quartiers, l'insertion urbaine de longue date des habitants permettent de penser que la rationalité urbaine doit être évaluée à l'aune d'un modèle transitionnel, de compromis, d'une construction de sens originale. Donc la culture urbaine en création dans ces quartiers doit se comprendre aussi en fonction de contraintes économiques et de transformations du système productif du bâti que la ville arabe traditionnelle ou le village pré-industriel ignoraient. » (Deboulet A., Vers un urbanisme d'émanation populaire, compétences et réalisations des citadins, l'exemple du Caire, Thèse de Doctorat 1994, Institut d'urbanisme de Paris, Université Paris XIICréteil, 729 p., p. 549).

22. Fanchette S., «Densités de population et urbanisation de l'espace rural : le cas du delta du Nil », Tiers-Monde, tome XXXI, n 121,1990, p. 29-56.

23. Cité par Lefebvre H., La pensée marxiste et la ville, Casterman/Poche, coll. mutationsorientations, 1972, p. 91.

24. Weber M., La ville (éd. allemande : 1947), Aubier, 1982,218 p. En 1978, Nwokolo I. B. (op. cit.) explique encore que « dans les villes du Moyen-Orient, le vaste hinterland rural génère une forte influence socio-culturelle qui fonde leur particularité et leur structure écologique unique »... Cf.Leca J-, «Individualisme et citoyenneté », Sur l'individualisme, éd. P. Birbaum. J. Leca, PFNSP, 1984. p. 159-209 ; Benet F., "The Ideology of Islamic Urbanization», International Journal of Comparative Sociology $n^{0} 4.1963$, p. 211-26 ; BAER G., « The Beginnings of Municipal Govemment in Egypt », Middie Eastern Studies, t. IV, $\mathrm{n}^{0} 2$, 1968, p. 118-40. La persistance de cette dimension s'explique aussi en raison du parcours des anthropologues qui ont suivi les migrants des campagnes vers les villes avec leur background de «ruralistes » (Cf. Foster G. M., Kemper R. V., «Introduction: a Perspective on Anthropological Fieldwork in Cities », Anthropologists in Cities, Boston, 1974, p. 1-17).

25. Abu Lughod J., Cairo, 1001 Yearsorthe City Victorious, 1971,284 p.

26. Franck M., Quand la rizière rencontre l'asphalte, semis urbain et processus d'urbanisation à Java-est, Études insulindiennes/Archipel $\mathrm{n}^{0} 10$, EHESS, 1993, $282 \mathrm{p}$.

27. El Kadi G., «La division sociale de l'espace du Caire : ségrégation et contradictions », op. cit. ; du même auteur, L'urbanisation spontanée au Caire, Fascicule de recherches $n^{\circ}$ 18, Orstom/Urbama, Université de Tours, 1987 ; voir également Mahmoud F. M., "Effect of Slum Areas on SocioEconomie DevelopmenI of Cairo City ", Population and Development Research, Monograph Series ${ }^{\circ}$ 1, Cairo Demographic Center, 1994, p. 334-340.

28. «'Ashwaiyyâl al-kubâr ", al-Ahrâm al-lqtisâdî, 16/01/95, p. 14-16. 
29. Deboulet A., Vers un urbanisme d'émanation populaire, compétences et réalisations des citadins, l'exemple du Caire, op. cit. : "En Égypte, la densification constitue la règle. Cela est très positif et évite le gaspillage accru de terrains, notamment agricoles, mais en retour, il faut bien voir que c'est à ce stade que se produit la perte de contrôle sur la gestion de l'espace local. Certes, les voisins continueront dans l'ensemble à se connaître, mais on perçoit déjà, et Dar al-Salâm en constitue un bon exemple, que la vigilance collective décroit. Il semble en fait surtout que les habitants ne sont de taille à négocier qu'avec des résidants de conditions économiques sensiblement égales. » (p. 726) Cela renvoie aux formes d'organisation sociale caractérisant la ville qu'identifiait Louis Wirth (1938, op. cit.), notamment à « l'érosion des bases traditionnelles de la solidarité sociale ».

30. Volait M., 1988, op. cit. ; El Kadi G., «Le Caire : la ville spontanée sous contrôle ». Maghreb Machrek. numéro spécial, 1994, p. 30-41.

31. El Kadi G., 1994, op. cit.

32. Panerai P., «Sur la notion de ville islamique » (Yémen, Sanaa), Peuples méditerranéens $n^{\circ} 46$, 1989, p. 13-33.

33. Ce processus de dégradation-dédensification du centre, avec insertion de foyers de modernisation très localisés, se retrouverait, selon des temporalités comparables, à Bombay, où les effondrements d'immeubles sont aussi fréquents qu'au Caire (cf. Charbogne M. B., « Bombay. Une gestion immobilière génératrice d'exclusion », Bulletin du Cera ${ }^{\circ} 39$, Presses Universitaires de Caen, 1994, p. 11-24), ou encore à Paris entre 1870 et 1945.

34. De même, entre 1980 et 1990 , les quatre circonscriptions centrales de Mexico ont perdu quelque 600.000 habitants, en partie à la suite du tremblement de terre de 1985 (Monnet J., « Mexico et les surprises du recensement ", L'Ordinaire n 130, Gral, Université de Toulouse, 1990). 35. En 1981, les urbanistes du GOPP et de l'IAURIF estimaient, à 340 ha par an la surface perdue pour l'agriculture (GOPP-IAURIF. Long Range Urban Development Schema). Si l'on estime que la surface urbanisée occupe moins de $50 \%$ de la surface des communes, ces données se tiennent. En 1993, à Gîza, dans les zones informelles les plus denses, 30 \% des parcelles n'étaient pas bâties (GOPP-IAURIF, Giza, Desert Development and Informal Area Planning). Autre source: selon une publication récente de l'Onu, la consommation de terre cultivable serait passée de 330 ha durant la période 1966-77 à 590 ha durant la période 1977-82. L'extension des villages serait responsable de $40 \%$ de ce changement d'usage du sol, les activités industrielles de $28 \%$ et l'étalement de l'agglomération de 38 \% (Onu-Centre for Human Setttements, Metropolitan Planning and Management in the Developing Worid: Spatial Decentralization Policy in Bombay and Cairo, Nairobi, 1993,155 p.). Entre 1986 et 1989, selon une étude conduite par le Remote Censing (Le Caire), la consommation d'espace en cultures serait tombée à 150 ha par an.

36. $\mathrm{L}^{\prime}$ indice de concentration peut être noté de la façon suivante : $I C=1 / 2 \Sigma^{n} \mid\left(\mathrm{x}_{\mathrm{ij}} / \mathrm{X}_{\mathrm{j}}\right)-\left(\mathrm{X}_{\mathrm{i}}-\mathrm{X}_{\mathrm{ij}} / \mathrm{X}\right.$ $\left.--\mathrm{X}_{\mathrm{j}}\right)$ - avec $\left(\mathrm{x}_{\mathrm{ij}} / \mathrm{X}_{\mathrm{j}}\right)$ exprimant le rapport d'un groupe d'une des $\mathrm{n}$ zones sur la population totale de ce groupe ; - et $\left(\mathrm{X}_{\mathrm{i}}-\mathrm{x}_{\mathrm{ij}} / \mathrm{X}-\mathrm{X}_{\mathrm{j}}\right)$, le rapport des groupes restants (total de la zone moins le groupe pris en compte) sur le total de la population active définie, moins le groupe cible.

37. Cf. Carte de Khânka 1/25.000e, couverture 1993. Misâhat al-'Askariyya, nº NH36.

38. Ireton F., Denis E., « Note sur les échanges migratoires entre le Grand Caire et la province et sur les migrations dans l'aire métropolitaine », Lettre de l'observatoire urbain du Caire contemporain $n^{\circ} 36$, Cedej, 1994, p. 32-41.

39. Bayhum N., David J.-C., « Espaces du public et du négoce à Alep et à Beyrouth », Les Annales delà recherche urbaine, $\mathrm{n}^{\circ}$ 57-58,1993, p. 191-205.

40. $f t=0,8$; taux de croissance annuel, population totale $2,99 \%$, population active $1,81 \%$.

41. Cf. Dupont V., Dureau P., «Rôle des mobilités circulaires dans les dynamiques urbaines, illustrations à partir de l'Equateur el de l'Inde », Tiers-Monde, t. XXXV, n 140,1994.

42. Pierre Veltz, dans la lignée des travaux visant à définir les métropoles ou les «villes globales» (Saskia Sassen) distingue trois effets métropolitains de première importance: la 
"productivité des interfaces », la «vitesse d'apprentissage » et l'« assurance-flexibilité ». Voir «Les ressorts de la métropolisation », Le Débat n 80, mai-août 1994, p. 188-189.

43. Cf. Burgel G., La ville aujourd'hui, Hachette, 1993, $220 \mathrm{p}$.

44. Ghitany G., Épître des destinées, Seuil, 1993.

45. Le coefficient de spécialisation peut être calculé de la façon suivante : $\mathrm{CS}_{\mathrm{i}}=\Sigma_{\text {jem }} \mathrm{I}\left(\mathrm{x}_{\mathrm{ij}} / \mathrm{x}_{\mathrm{i}}\right)-\left(\mathrm{X}_{\mathrm{j}} /\right.$

X)I. Cf. Petsimeris P., «Le Grand Londres entre polarisation sociale et gentrification ", Au centre des villes. Dynamiques et recompositions, éd. J. Chevalier, L'Harmattan, coll. Géographie sociale. 1984, p. 193-212.

46. Perrot M., "La ville et ses faubourgs au XIXe siècle », Citoyenneté et urbanité, éd. Esprit, 1991, p. 65-83.

47. Panerai P., Noweir S., «Du rural à l'urbain», Égypte/Monde arabe $\mathrm{n}^{0} 1,1990, \mathrm{p} .97-124$ ("Quelques observations, cependant, indiquent les voies d'une autre recherche où l'étude de l'informel et du découpage agricole éclaire, à n'en pas douter, celle de la ville ancienne. ») El Kadi G., L'urbanisation spontanée au Caire, op. cit.

48. Singerman D., Avenue of Participation. Family, Politics and Networks in Urban Quarters of Cairo, Princeton University Press, New Jersey, 1995, 335 p.

49. Berque J., op. cit., p. 86 ; Denoix S., Décrire Le Caire. Fustât-Misr d'après Ibn Duqmâq et Maqrizî, IFAO, Le Caire, 1992, 159 p.

50. Levy J., 1994, op. cit.

51. II peut être noté de la façon suivanle: $Q L_{j^{\prime}}=\left(x_{i j} / X_{i}\right) /\left(X_{j} / X\right)$.

INDEX

Mots-clés : urbanisation, Le Caire, ville, géographie

\section{AUTEUR}

\section{ERIC DENIS}

Cedej-CNRS 